\title{
17 \\ Tool Use and Manufacture in the Last Common Ancestor of Pan and Homo
}

\author{
CAMPBELL ROLIAN and SUSANA CARVALHO
}

\begin{abstract}
Cumulative culture, including complex forms of tool use, is often seen as a defining feature of hominins, but the manufacture and use of tools are by no means restricted to humans and their ancestors. Although tool use is a rare behavioral strategy in the animal kingdom, the use of tools for both subsistence purposes (i.e., extractive foraging tasks) and aggressive display is well documented in other primates, and in other vertebrates (Shumaker et al. 2011). Among nonhuman primates, chimpanzees have been the focus of numerous studies reporting on the regional variation, sometimes labeled cultural, in the behavioral repertoires of different groups (Whiten et al. 1999; Carvalho and McGrew 2012). After humans, chimpanzees (Pan troglodytes) have the largest repertoires of tool use and manufacture known among primates (McGrew 2017). Bonobos (Pan paniscus) have been the focus of much less research, and appear to be infrequent tool users in natural contexts (Whiten et al. 1999; McGrew 2004; Haslam 2014; for in-depth comparisons between Pan troglodytes and Pan paniscus and their tool use, see Koops et al. 2015; Gruber and Clay 2016). Because chimpanzees and bonobos are both our sister species and, notwithstanding their different degrees of reliance on technology, both are known to use tools, a comparative study of tool behav-
\end{abstract}


ioral variation, but also of the morphological and cognitive correlates of tool use (e.g., hand morphology: Marzke 1997) between Homo and Pan may be useful in making inferences on the tools and tool-making behaviors of our last common ancestor, the LCA.

In this chapter, we will address two questions pertaining to tool use and manufacture in the LCA. Was the LCA able to use and / or manufacture tools? And if so, what type of tools likely composed the tool kit of this Miocene ape, and in which ecological contexts was it more likely to use tools to solve problems? To answer these questions, we will explore four complementary lines of evidence: (1) comparative tool use in extant apes and other primates, (2) comparative anatomy and biomechanics of primate hands, (3) comparative cognition in hominoids, and (4) evidence from the fossil and archaeological records of early hominins. It is not our intent to provide exhaustive reviews of each line of evidence, but rather to leverage current knowledge in each, especially with regards to the genus Pan, to paint the most likely picture of what tool use and manufacture in the LCA would have looked like.

We will limit our discussion primarily to tool use in the wild. Tool manufacture and use in captivity is well documented for a number of primates that have not been observed to do so in the wild (reviewed in Shumaker et al. 2011). However, captive settings lack ecological validity, making it difficult to evaluate the roles of group size, kinship, food availability, and other factors in understanding the emergence and duration of novel technological behavior.

\section{What Is a Tool?}

Before answering the questions outlined above, it is important to define what we consider to be tools in the context of primate behavioral ecology. We define a tool as a noncorporeal, unattached object that an animal makes and / or uses to modify itself, another individual, or its proximate environment (see also Shumaker et al. 2011). Tools may be deliberately modified objects (tool manufacture), or they may be objects used without prior modification-and modified only by use (tool use). Tools may be used in flexible, complex ways, sequentially, as part of tool composites or serving multiple functions. They are employed in multiple tasks or behaviors in the context of a primate's natural habitat and ecology. The most commonly reported uses of tools in 
wild primates fall into three categories: (1) tools used for extractive foraging and / or food processing, (2) tools used in grooming or increasing physical comfort/hygiene, and (3) tools used for communication.

\section{Tool Use and Manufacture in Nonhuman Primates}

\section{Chimpanzee (Pan Troglodytes)}

Apart from humans, tool use and manufacture is best documented in chimpanzees, who show variation in their technological repertoires-for example, sequential tool use and tool composite use that have yet to be observed in any other extant nonhuman primate (Carvalho et al. 2009; Carvalho and McGrew 2012). Over fifty-five years of observations in the wild, from multiple long-term sites in East, Central, and West Africa, have painted a detailed picture of the diversity and complexity of their tool kits and behaviors (Whiten et al. 1999; see McGrew 2017 for an update on long-term sites). Unlike all other tool-using nonhuman primates, there is no well-studied chimpanzee population for which tool use has not been reported. As with humans, technology is present independently from habitat type, group size, predation pressure, and availability of resources. Every few years, a previously unknown type of tool or tool behavior is reported for specific chimpanzee populations (see, e.g., Sanz et al. 2004; Pruetz and Bertolani 2007; Koops et al. 2010, Lapuente et al. 2016; Musgrave et al. 2016 for recent examples). A thorough discussion of each tool, its use(s), and contexts is beyond the scope of this chapter, and several volumes have been written on the subject (see, e.g., McGrew 1992, 2004; Shumaker et al. 2011). Here we will limit our discussion to a few key features of chimpanzee tool behavior that make it unique among nonhuman primates.

Chimpanzees habitually use many different tools for communication with conspecifics, and/or as deterrents against perceived predators (or human observers) (Matsuzawa 1999). Tools used in this context include branches shaken, waved, or dropped from trees, objects (e.g., stones) thrown as part of conspecific displays (e.g., male-male aggression), and leaves that are ripped or torn to produce sounds that may be involved in courtship (Whiten et al. 1999). Tools are also used for grooming or personal comfort. These include leaves used as napkins or as sponges (for drinking), and 
branches used to swat away insects, or to scratch an itch (Goodall 1986; Sanz and Morgan 2007).

The most complex and diverse tools in chimpanzees are used for extractive foraging, for example, the use of stone tools for nut cracking (Carvalho et al. 2008) or the sequential use of five different tools to obtain honey (Boesch et al. 2009). Chimpanzees are omnivores, and while the fruits that make up large portions of their diets do not typically require tools to consume, many other consumed foods require tool-assisted extraction or processing. Sticks and twigs are among the most common foraging tools used by chimpanzees. Sticks of varying diameters are used to probe holes in insect mounds, to pry or dig them open, and, most famously, to dip the sticks in these mounds and extract termites and other insects (aka "fishing") (e.g., Goodall 1963; McGrew 1974; Sanz et al. 2004). Using sticks to extract honey has also been documented from multiple long-term study sites (e.g., Boesch et al. 2009; Sanz and Morgan 2009). Savanna chimpanzees at Fongoli, Senegal, have also been observed to use wooden spears to hunt for bushbabies (Pruetz and Bertolani 2007).

Habitual lithic technology has been observed only in West African chimpanzees, especially at Taï, Ivory Coast (Boesch and Boesch-Achermann 2000), and Bossou, Guinea (Fushimi et al. 1991; Carvalho et al. 2008, 2009), and constitutes a tiny proportion of the tool-use repertoire. The most common use of stone tools is as hammers, which are used to crack open the nuts of several tree species (Figure 17.1). On occasion, and only at Bossou, another large yet movable stone is used as an anvil upon which the nut is placed, and smaller stones have also been used by chimpanzees at that site to prop up and stabilize the anvil. Experiments in a "natural laboratory" setting at Bossou, in Guinea, show that the chimpanzees discriminate features of stone tools when selecting hammers or anvils, and often transport and reuse them for nut cracking at later times or places, before ultimately discarding them (i.e., "future planning": see Rosati, this volume; Carvalho et al. 2008; Carvalho and McGrew 2012). Moreover, there is regional variation between the sites in these operational sequences (chaînes opératoires) that maps onto local environmental factors (e.g., the availability of raw material), leading to differences in the stone assemblages left behind (Carvalho et al. 2008; Koops et al. 2013). This sort of cognitive flexibility related to lithic tools has not been documented in any other primate except for humans and their hominin ancestors. 


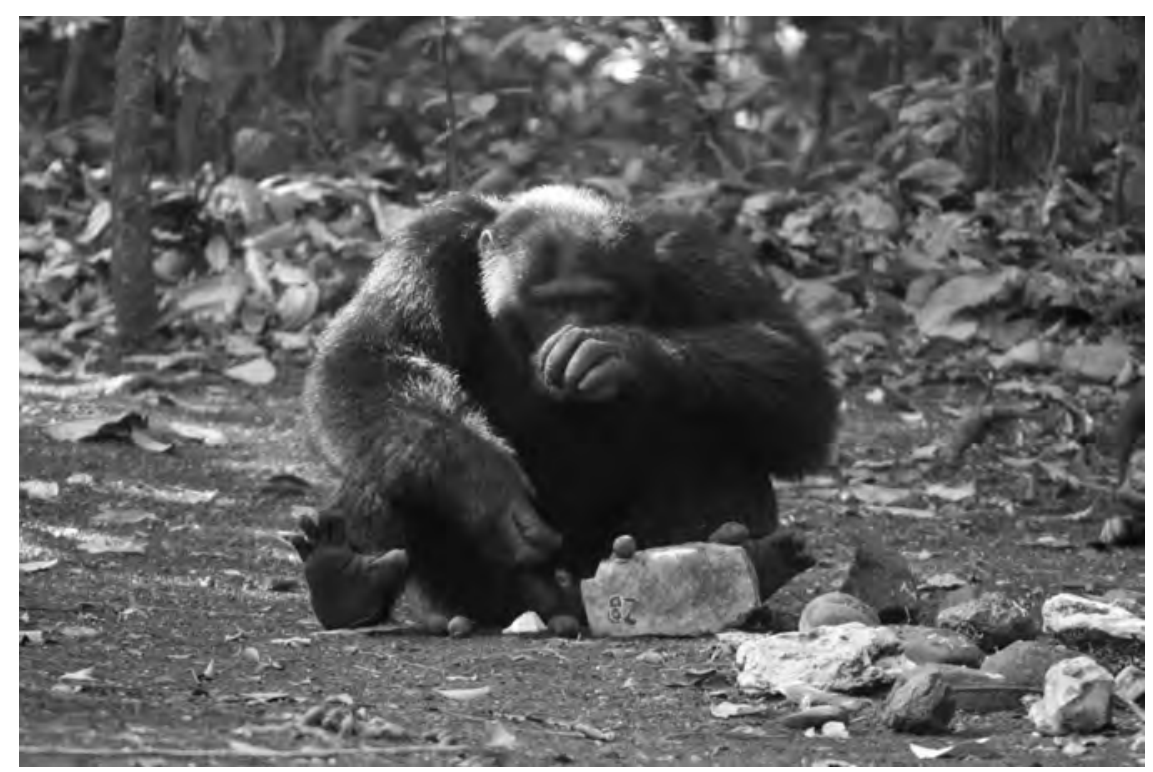

FIGURE 17.1. Adult male in the outdoor laboratory of Bossou, Guinea, using a pair of stones-anvil and hammer-to crack open nuts (Elaeis guineensis and Coula edulis sp.). Photo: Susana Carvalho.

In addition to their frequent tool use, wild chimpanzees are also accomplished tool makers. Many of the organic tools described above require one or more steps to make. The simplest form of manufacture involves removing a branch from a tree, in a single step, to use as a signaling device (see below). Manufacturing more complex tools, especially for extractive foraging, typically requires greater modification of the raw materials before use. For example, dipping sticks may be stripped of leaves or bark before they are used to fish for insects. In a further elaboration of this activity, Sanz et al. (2009) reported that chimpanzees at Goualougo, Republic of Congo, deliberately fray the tips of the stick, turning it into a brush that is more efficient for extracting ants (Whiten 2011).

In contrast to these organic tools, the stone tools used by West African chimpanzees are not deliberately manufactured. Although they are used together (hence are a composite tool; see below), the hammers and anvils are unmodified stones that are modified only by the use (Figure 17.2). On two occasions, Carvalho et al. (2008) observed that stones were unintentionally fractured as part of the nut cracking process. In one case, a large flake was 


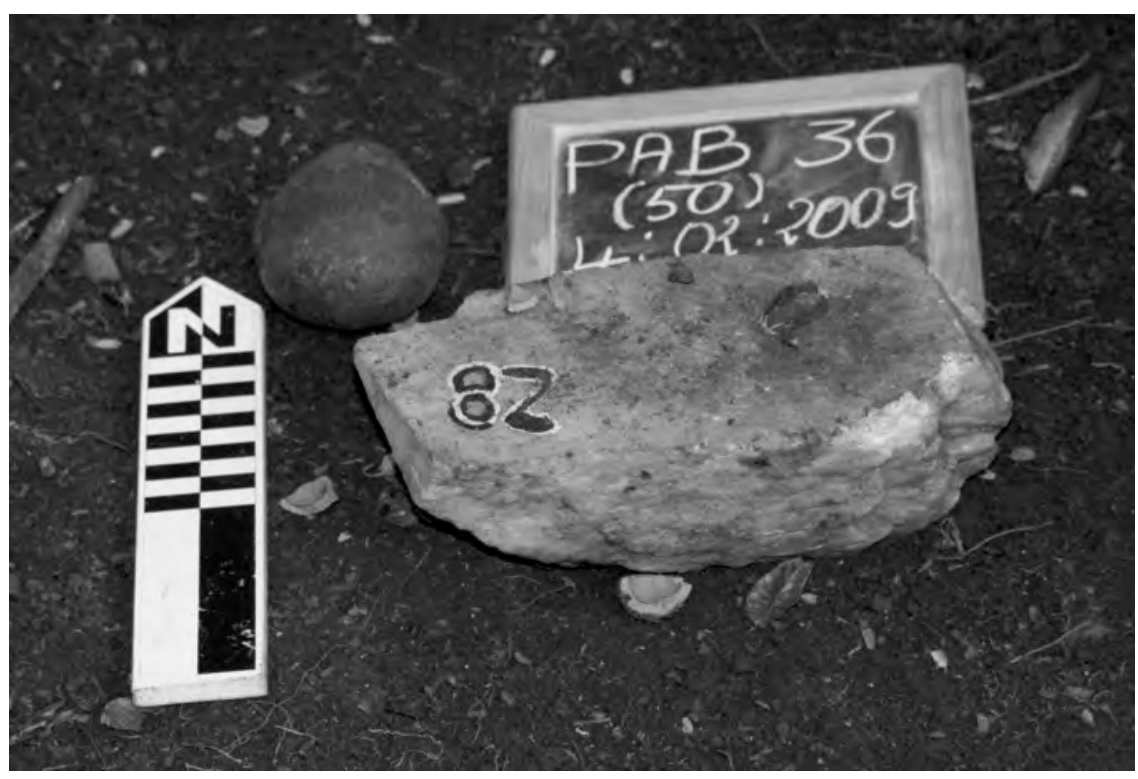

FIGURE 17.2. Pounding tools after being used by the subject in Figure 1 during one experimental nut-cracking session. Note the damage on the edges of the anvil as a result of missed strikes-depending on the type of raw material used, this missed blow may produce objects that are very similar to human-produced flakes. Photo: Susana Carvalho.

detached from the anvil, and the user subsequently attempted to use the detached flake as a hammer, while the fractured tool was discarded, making this one of the few reported cases of the reuse of a by-product of a percussive activity by nonhuman primates.

Tool use and manufacture have been documented in every chimpanzee population studied to date, and hence is considered to be universal (and likely plesiomorphic) among chimpanzees (McGrew 1992, 2004). Still, the wealth of data accumulated over the last fifty years from multiple long-term study sites shows that there is substantial local variation in tool repertoires and behaviors among populations (reviewed in Whiten et al. 1999). Some tools and behaviors are universally shared among all documented populations, such as dragging, shaking, or waving branches in conspecific communication, probing with sticks, or using leaves as sponges (Whiten et al. 1999). Others may be absent from particular sites for ecological reasons-for example, the absence of a particular food source or a low probability of encountering the resource in the home range (Koops et al. 2013). 
Many tools, however, are unique to specific populations, though ecological factors do not explain their absence elsewhere. This is exemplified by the stone tools, which are only found in West Africa, despite the availability of suitable stones and nuts at sites in Central and East Africa (Whiten et al. 1999; but see Koops et al. 2014 for an opposing view on the "method of exclusion," which identifies culture in wild animals by excluding ecological and genetic factors to explain geographical variation in behavior). This suggests that tool traditions have evolved locally among chimpanzees at the subspecies and population levels. More broadly, it also indicates that tools and tool behaviors have the potential to evolve rapidly, and in parallel, among populations and even species. This has important implications for reconstructing tool behavior in the LCA, as it suggests that convergent evolution (homoplasy) of technological behavior is likely, or even frequent, and thus, although not mutually exclusive with homoplasy, parsimony-based arguments in favor of the presence of tool behaviors in the LCA must be examined critically (see below).

The rate of evolution of these behavioral traditions may relate to the pathways of transmission of information in chimpanzees. There is little doubt that social influences are important in the process of acquisition and diffusion of tool use in primates (Biro et al. 2003; Lonsdorf et al. 2004; Liu et al. 2011; Hobaiter et al. 2014). The process of learning a tool behavior has been described as "Education by Master-Apprenticeship" (Matsuzawa et al. 2001), in which learning is facilitated by tolerant conspecifics allowing juveniles to closely observe their behavior during extended periods of time (see also Henrich and Tennie, this volume). In the absence of active teaching (but see Musgrave et al. 2016 for a potential exception), a combination of trial and error, with the recycling by youngsters of tools made or used by adults (Biro et al. submitted), combined with high levels of tolerance, would likely favor the emergence and endurance across generations of more complex tool behaviors.

\section{Bonobos (Pan Paniscus)}

Tool use and manufacture has also been documented in wild bonobos, but it has been described as very rare (Gruber and Clay 2016). Although this may reflect the paucity of bonobo field studies compared with chimpanzees (McGrew 2010), the factors that best explain the major differences in tool use be- 
tween chimpanzees and bonobos have been identified as one of the most urgent questions to address in future Pan behavioral studies (Gruber and Clay 2016). Bonobos do not use tools for extractive foraging, but have been seen using tools for communication and personal grooming. Much like chimpanzees, bonobos have been observed to use modified branches for displays, to swat away flies with leafy twigs, and to use moss sponges to absorb water (Hohmann and Fruth 2003). Probing tools and behaviors, and more specifically insect fishing, have never been observed directly in wild bonobos, though Badrian (1981) reports indirect evidence of this behavior from Lomako Forest (Democratic Republic of Congo) in the form of broken termite mounds with nearby harvesting sticks (see also McGrew et al. 2007).

Until very recently the use of stone tools had not been observed for bonobos outside of captivity. Neufuss et al. (2017) recently reported on the nut-cracking behavior of wild-born, rehabilitated bonobos in the Lola ya Bonobo sanctuary in the Democratic Republic of the Congo. Although not free-ranging, the bonobos live in a thirty-hectare natural environment, with access to both high canopy forest areas with palm oil trees, as well as more open areas. The bonobos engaged in palm nut cracking behaviors frequently, using a variety of hand grips (see below) that matched the varying sizes and weights of the hammerstones at their disposal. The authors also noted that individuals selected the most appropriate stones and hand grips to improve the efficiency of the nut cracking (i.e., the number of hits required to crack a nut and the number of nuts per minute), matching the efficiencies previously observed in chimpanzees (Biro et al. 2006; Neufuss et al. 2017). It remains to be seen whether the behavior exhibited by these semi-wild bonobos is also found in the wild, or whether their use of stones for nut cracking is a technological innovation acquired more recently in the sanctuary. Nonetheless, this shared ability to perform stone tool cracking behaviors in chimpanzees and bonobos indicates that this is likely a plesiomorphic behavior for the genus Pan, with implications for its presence in the LCA (see below).

\section{Other Hominoids}

Breuer et al. (2005) reported the only two, anecdotal, cases of western gorillas (Gorilla gorilla) using tools in the wild. One adult female was observed using a stick to gauge the depth of water in an elephant pool. In the second case, another adult female detached the trunk of a small shrub and used it to 
support and stabilize herself as she dredged food from a swamp. She then used this trunk as a bridge upon which she walked bipedally to cross an area of the swamp. It is noteworthy that in these individuals, the tools were not used directly for extractive foraging, but rather to assist them in adapting to an atypical environment. As the authors note, the fact that tool use has rarely been documented in wild gorillas may be precisely because the exploitation of their primarily food resources (e.g., piths, leaves, fruit) involves extracting or processing that can be accomplished by hands and/or teeth alone (Breuer et al. 2005). However, a juvenile female mountain gorilla in Volcanoes National Park (Rwanda) was also recently observed using a stick as a probe to extract ants from their nest (Kinani and Zimmerman 2015).

Wild orangutans are frequent tool makers and users (Meulman and van Schaik 2013). As in chimpanzees, tools are used in different contexts, including extractive foraging (e.g., using sticks as chisels to pierce the shell of durian fruit), communication with conspecifics (e.g., branch dragging displays), and as a means of increasing physical comfort (e.g., use of leaves as napkins, shelter against sun/rain). In contrast to chimpanzees, the tool kits of orangutans do not include inorganic materials, or at least their use has not yet been documented in the wild-which may be linked with the degree of arboreality of this ape, less exposed to the opportunity to encounter and manipulate lithic objects. Cultural variation in tool use across orangutan subspecies, and across different sites, is well documented (van Schaik et al. 2003). In fact, very few tools and tool behaviors are universally shared among different populations of Bornean and Sumatran orangutans, and none of these relate to extractive foraging (van Schaik et al. 2003). This suggests that, as in chimpanzees, local ecological conditions, especially the availability of food resources that require extractive processing, play an important role in the nature, diversity, and frequency of tools and tool use in these omnivorous primates.

Gibbons have been observed on occasion to use branches as tools, in the context of displays and communication with conspecifics (Carpenter 1940; Deng and Zhou 2016). To date, however, they have not been observed to use tools for extractive foraging purposes in the wild (van Schaik et al. 1999). The absence of tool use in these primates may be attributed at least in part to their diet, which consists mainly of easily accessible fruits (e.g., figs) and leaves (Palombit 1997). Although insects may make up a substantial portion of some species' diets as well (e.g., Fan et al. 2009), this appears to be opportunistic 
or seasonal, and gibbons do not feed on insect species that require extractive techniques, such as termites.

\section{Other Primates}

1. Old World Monkeys: Tool use among wild cercopithecoid monkeys has been documented in baboons and macaques. In The Descent of Man (1871), Darwin reported secondhand on observations of chacma baboons using stones as defensive tools. Specifically, the baboons would drop or roll stones down the rocky walls of the canyons they inhabit onto predators below. These anecdotal reports were later confirmed by Hamilton and colleagues (1975), who observed three separate baboon troops engaging in this behavioraimed at them-in a desert floor canyon in Namibia. Tool use in extractive foraging contexts in the wild has not been documented for papionins. Among macaques, semi-wild pig-tailed macaques (Macaca nemestrina) have been observed using leaves to wash dirt/mud off seeds and fruit before eating them (Chiang 1967). In addition, long-tailed macaques in Thailand (Macaca fascicularis aurea) have been observed to use unmodified stones to pound and crack open the shells of oysters (Malaivijitnond et al. 2007; Gumert et al. 2009; Haslam et al. 2016a). These authors report that the handheld stone tools were occasionally transported from one activity site to another. Thus, long-tailed macaques are one of only two nonhominoid species known to engage in percussive tool use in the wild, albeit this behavior seems to be restricted to a few groups living in the coastal areas of Thailand.

2. New World Monkeys: The most commonly reported use of tools is in wild capuchin monkeys (especially bearded capuchins, Sapajus libidinosus) (reviewed in Ottoni and Izar 2008). Specifically, capuchins are proficient nut crackers, habitually using pounding stones and anvils to crack open palm nuts (Visalberghi et al. 2007), and have seemingly been doing so for at least seven hundred years (Haslam et al. 2016b). Capuchins are selective in the type and size of rock they use as a hammer, and have been observed to transport these to different anvils around their home ranges (Visalberghi and Fragaszy 2013). Recent evidence also suggests that capuchins deliberately break stones using stone-on-stone percussion, a behavior that accidentally produces flakes with attributes (e.g., conchoidal fracture, bulb of force, platform, weight, sharp edges) that are indistinguishable from intentionally produced flakes from early hominin stone tool assemblages (Proffitt et al. 2016) 


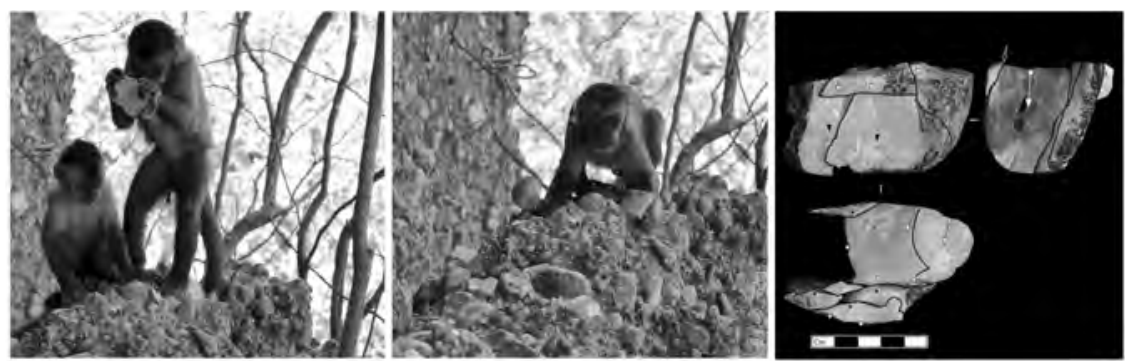

FIGURE 17.3. Stone-on-stone percussive behavior in capuchins (Sapajus libidinosus). In the left panel, a juvenile observes as the adult prepares to strike the rocky conglomerate with the stone hammer. In the middle panel, the hammer shatters into several pieces. Some of the flakes that resulted from the percussive event are shown in the right panel. Adapted by permission from Macmillan Publishers Ltd.: Nature (Proffitt et al. 2016. Wild monkeys flake stone tools. Nature 539: 85-88), Copyright 2016.

(Figure 17.3). The functionality of this behavior is not well understood, but the fact that the capuchins lick the rock each time it fractures raises the possibility that they are getting trace minerals from the rocks. This is an excellent example of a process by which flakes are produced (albeit apparently not used, hence they are not "tools" sensu strictu) that has not been considered in previous analyses of stone tool assemblages. There is also marked geographical variation and limitation in the habitual use of percussive technology in bearded capuchins, with mostly those inhabiting savanna environments exhibiting these behaviors. It has been suggested that increased terrestriality may be partly responsible for this variation (see below; Meulman et al. 2012; Visalberghi and Fragaszy 2013).

3. Prosimians: Strepsirrhine primates do not use or make tools, either in the wild or in captivity (Santos et al. 2005).

\section{Hand Anatomy, Dexterity, and Tool Use in Modern Primates}

Tool use and manufacture among primates is predicated on their ability to manipulate and/or modify objects for specific tasks with their hands. In other words, hands are the "hardware" that make primate tool use possible. Hand anatomy and morphology vary substantially among primates (Napier 1980). Hence, there has been a long-standing interest in correlating variation in hand morphology and dexterity among primates with interspecific vari- 
ation in the ability to make and use tools (van Schaik et al. 1999). Our survey of tool use among nonhuman primates clearly shows that a specific hand morphology, by itself, is not a prerequisite for tool use. However, hand morphology likely influences the nature of the tools that can be made or how they are used. Hence, comparing hand anatomy among chimpanzees and humans, especially as it relates to dexterity, can yield insights into the hand anatomy of the LCA and, by extrapolation, into its ability to make and use tools.

\section{Primate Hand Grips}

John Napier (Napier 1956) first proposed a classification system for hand grips in primates based on a dichotomy between power grips and precision grips. Power grips are those in which the object, be it a tool or an arboreal support, is pinched by the fingers and stabilized across the palm. The thumb is more or less involved in power grips. As the name implies, power grips are used when large muscle forces are necessary to hold or manipulate objects. In contrast, precision grips involve mainly the digits, as opposed to the palm. Precision grips involve opposition of part(s) of the thumb to the other digits. Napier argued that humans performed better at precision grips, while other primates relied more on power grips for manipulation and locomotor behaviors. There is likely more of a continuum between these grips, and all primates are capable of using both to variable extents (Marzke 1997).

Additional analyses of manipulative behavior in primates, especially in modern humans replicating stone tool use, have refined this grip typology. Marzke and colleagues' studies of manipulative behavior in humans and nonhuman primates (e.g., Marzke and Shackley 1986; Marzke et al. 1992) found that when archaeologists experimentally replicate stone tools, they mainly use three types of precision grip. In the "three-jaw chuck," the palmar aspect of the thumb opposes a variably flexed third digit, and the index rests atop and stabilizes the object, which is partly buttressed against the second metacarpal. This grip is primarily used for holding hammerstones during hard-hammer percussion. The "two-jaw chuck" opposes the distal thumb pad to the side of the index, that is, as one would hold a key. This grip is often used in handling stone flakes for more delicate tasks. Finally, cores were often manipulated using "cradle grips," in which the thumb and four fingers firmly pinch and maneuver large stone cores, exposing surfaces for flake removal by the hammerstone (Marzke 1997) (Figure 17.4). 

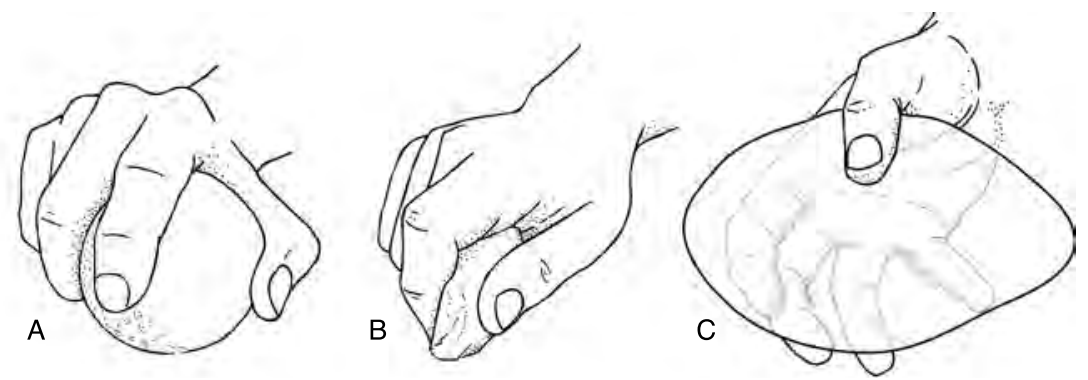

FIGURE 17.4. Precision grips used to wield Oldowan tools. (a) Three-jaw chuck grip used to hold a hammerstone. (b) Key pinch grip used to hold a flake. (c) Cradle five-jaw grip used for large cores. Modified from Rolian et al. (2011), with permission from Elsevier.

One feature these three grips have in common is that they require forceful and sustained opposition of the thumb to the radial digits, either to make a stone flake or to use it (Marzke 2013). By extension, the ability to wield and make stone tools may thus be directly linked to the user's ability to forcefully oppose the thumb to the lateral digits. If it also has correlates in hand musculoskeletal anatomy, and these can be traced in the fossil record or inferred in the LCA, then it may provide additional information on the types of tools the LCA would have used.

\section{Chimpanzee Hand Anatomy and Grips}

Unlike humans, the hands of chimpanzees are used in locomotion, as well as to manipulate objects. The chimpanzee hand may thus reflect an evolutionary compromise between selective pressures related to manipulation, if any, and those related to locomotion. One of the most salient features of the chimpanzee hand skeleton is its digit proportions, specifically the ratio of thumb length to finger length (also known as intrinsic hand proportions). After orangutans, chimpanzees have the lowest ratio among hominoids, and one of the lowest among all primates (Marzke 1997; Almecija et al. 2015; Pilbeam and Lieberman, this volume). The thumb is also relatively gracile (Rolian et al. 2011). In addition, its proximal phalanges are highly curved in the dorsopalmar plane. This feature is also prominent in gibbons and orangutans, as well as a number of fossil hominoids (see below). The degree of phalangeal curvature likely relates to the biomechanics of below-branch, or- 
thograde suspension and / or brachiation, although it may be largely epigenetic (Richmond 2007).

As in all hominoids, the thumb is opposable, though the articular facet between the thumb metacarpal and the trapezium is strongly curved (saddleshaped), limiting its mobility but increasing its stability (Tocheri and Marzke 2007; Tocheri et al. 2008). The distal phalanges have narrow apical tufts (Mittra et al. 2007). In terms of pertinent arm and hand muscles, chimpanzees have powerful elbow, wrist, and digital flexors, the latter reflected in strong flexor sheath ridges on the phalanges of the fingers (Thorpe et al. 1999). In contrast, the intrinsic muscles of the hand, and in particular those that support the thumb (thenar muscles), are poorly developed (Marzke 1992).

The sum of these morphological features indicates that the chimpanzee hand would not perform well in manipulative tasks that require the forceful opposition of the thumb to the other digits. In fact, such forceful, singlehanded precision grips have not been observed in the wild (Marzke 2013). This further suggests that chimpanzee hands are constrained in their ability to make and use stone tools, which requires firm grips. The most significant limitation on this ability is not its short thumb relative to long fingers. Most primates, including chimpanzees and Miocene apes (Lovejoy et al. 2009b; Almecija et al. 2012), are able to oppose the digit tips in a fine precision grip, but such grips are not useful for manipulating stone tools (Marzke and Shackley 1986).

A more significant constraint on the ability of chimpanzees to produce the forceful precision grips required for stone tool use stems from their relatively weak intrinsic hand muscles and gracile thumbs. Using an experimental biomechanics approach, Rolian et al. (2011) determined that the total muscle force potential necessary to stabilize the fingers and thumb joints during simulated Oldowan stone tool use exceeds the available force potential of the chimpanzee intrinsic muscles of the hand. Moreover, even if chimpanzees were physically able to wield hammerstones and flakes in the way modern humans can, the relatively gracile thumbs and overall smaller joint surfaces in the carpus and thumb metacarpal would produce large joint stresses under the kinds of loads required to wield stone tools.

Given these considerations, it is perhaps not surprising that the tool kit of chimpanzees and bonobos is limited largely to tools that do not require the forceful opposition of the thumb to lateral digits to produce or use, such as twigs and leaves, or conversely large-diameter branches and other objects 
(e.g., stone pestles) that are held using power grips, that is, with the objects buttressed against the palm. As discussed above, chimpanzees do use unmodified stone tools, albeit not universally. Importantly, however, the grips that chimpanzees employ to use hammers and anvils are not forceful precision grips. They are full-fledged power grips, in which the bulk of the force produced by smashing nuts is distributed across the palm(s). These grips do not involve the thumbs in forceful opposition to the other digits, but rather in the same plane as the fingers (Boesch and Boesch 1993). Similarly, the vast majority of grips used by wild bonobos are power grips, although forceful precision grips involving the thumb and lateral digits without the palm were occasionally used by two individuals in nut cracking (Neufuss et al. 2017).

\section{Human Hand Anatomy and Grips}

Several important features of the human hand are correlated with its enhanced ability to manipulate objects, and in particular to generate forceful precision grips. The thumb to digit ratio is the highest of all primates, reflecting not only an absolutely longer thumb, but also relatively shorter lateral digits. These proportions facilitate the opposition of the thumb to the tips of the other digits. The thumb bones are also robust, with larger articular facets, allowing joint reaction forces to be distributed over a greater surface area when loaded (Susman 1998; Rolian et al. 2011). In addition, the distal phalanges of the digits bear prominent apical tufts that underlie the division of the broad fleshy pulp of the fingertips into movable proximal and more firm distal compartments (Shrewsbury et al. 2003). A number of derived carpal shapes relate to increased range of motion of the wrist, increased opposability of the thumb and fingers, and increased internal stability between the carpal bones (reviewed in Marzke and Marzke 2000).

The human hand also has derived musculotendinous features that correlate with the ability to generate forceful precision grips. It has a fully separate and well-developed flexor pollicis longus, whereas in other apes it is absent or is still a part of the deep digital flexors of the digits (Linscheid et al. 1991; Marzke et al. 1999). Its intrinsic hand musculature is particularly well developed, both on the thumb (thenar) and fifth digit (hypothenar) sides. For example, the total physiological cross-sectional area of the thenar muscles in humans, which reflects the total force these thumb muscles can produce, is over twice as large as it is in chimpanzees (Marzke et al. 1999). 
This combination of short digits, a long robust thumb, and well-developed hand muscles is at least in part responsible for the ability of humans to generate and withstand large forces within the hand and fingers. Inasmuch as these forces are associated with lithic technology, and with stone tool manufacture in particular, they suggest that a specific hand anatomy is required to wield stone tools in the way that humans and their ancestors did.

\section{Hand Anatomy, Grips, and Tool Kits in the LCA}

The comparative morphological evidence suggests that Pan-like hand anatomy constrains the types of tools chimpanzees can manipulate, and the extent to which they can deliberately modify such tools. Thus, if we could directly examine the morphology of the LCA's hand, we might gain insight into the type of tools it used. Tocheri et al. (2008) used the principles of parsimony and the fossil record of hominin hand elements (see below) to reconstruct the most likely musculoskeletal anatomy of the LCA hand. They conclude that the hand of the LCA would have looked like that of an African ape. Specifically, they argue that skeletal features present among extant apes were also present in the LCA (i.e., they are homologies in the African apes), such as long fingers relative to the thumb, dorsopalmar curvature of the phalanges, marked ridges for the insertion of the digital flexor sheaths on the sides of the proximal phalanges. They also argue from parsimony that the thenar muscles would have had a relatively small cross-sectional area, and, as in African apes, there would have been no or a poorly developed flexor pollicis longus, but strongly developed digital flexor muscles.

Almecija et al. (2015) used a phylogenetically broad morphometric approach coupled with Ornstein-Uhlenbeck (OU) evolutionary modeling to reconstruct the ancestral states of digit lengths and proportions among hominoids. Using shape variables based on the ratio of metacarpal and phalangeal lengths to the cube root of body mass, they arrived at radically different conclusions from Tocheri et al. (2008): (1) the digital elongation of chimpanzees and orangutans is convergently derived, (2) human and gorilla proportions are plesiomorphic for great apes, and (3) the LCA's proportions are closer to this "plesiomorphic" exemplified by humans and gorillas, with "moderate" proportions between those of chimpanzees and modern humans. In other words, while its hands may not have looked like those of modern Pan, the LCA had intrinsic hand proportions capable of producing the precision grips 
associated with stone tool use, provided it also had well-developed hand muscles.

Almecija et al.'s (2015) conclusions, however, may have been influenced by their choice of characters used in the OU models, namely shape ratios of thumb and fourth digit element lengths to the cube root of body mass. Body mass is a common proxy for size; however, as Almecija et al.'s (2015) supplementary figure 4 suggests, hominoids have different linear relationships between body mass and digit length (with respect to their intercepts). For example, at comparable body sizes, Pan and Pongo fourth digits are absolutely longer than humans, while the reverse is true for gorillas. As a result, the reconstruction of ancestral hand extrinsic proportions in the LCA and in the last common ancestor of African apes and humans with short lateral digits and relatively long thumbs may have been driven at least in part by the low length-to-mass ratio of gorillas and humans. Put differently, even slight differences in the ratios obtained using different size proxies, such as skeletal measurements from the proximal limb skeleton, could lead to different LCA reconstructions, and hence to different interpretations of the relative evolutionary changes in each lineage (see, e.g., Pilbeam and Lieberman, this volume).

Based on this anatomical evidence, what sort of grips could the LCA produce? Its inferred hand anatomy is indicative of a hand that was capable of strong power grips that involve the fingers exclusive of the thumb. Similarly, the inferred proportions and muscle distribution suggest it would have performed as well as a modern chimpanzee (Tocheri et al. 2008), if not better (Almecija et al. 2015), in producing the forceful precision grips required to use and manufacture stone tools. Put simply, the inferred anatomy of the LCA hand suggests, if anything, a tool repertoire similar to modern chimpanzees, with mostly organic material that can be used with relatively little finger/thumb muscle force, and perhaps unmodified hammerstones and anvils.

\section{Cognition and Tool Use}

If hands are the hardware that facilitate tool use, then the brain is the operating "software" that directs the act of using and making stone tools. Though we cannot observe behavior or cognitive ability in the LCA directly, we can use comparative evidence and studies from modern primates to make infer- 
ences on the range of cognitive abilities required to use, and make, tools. Such studies reveal the existence, at least superficially, of a hierarchy of cognitive abilities associated with ever more complex tool kits and tool behaviors (e.g., Matsuzawa 1996; but see Shumaker et al. 2011 for a discussion of the myth that all tool behavior is "intelligent").

Perhaps the most basic cognitive requirement to make and use tools is an understanding of, and mental ability to correlate, cause and effect (i.e., causality, Urbani and Garber 2002). Many experiments in captivity indicate that most primates have a basic understanding of causality in the context of tool use and manufacture. These experiments usually take the form of a test that, if successfully completed, will lead to a food reward. Some of the experiments are specifically designed so that the reward cannot be obtained without using an object to modify the immediate environment of the individual, for example, using a stick to probe for food rewards that are otherwise inaccessible. By our definition, these are instances of tool use. These experiments are contrived, and do not necessarily reflect the ability of individuals (or species) to develop these skills in the wild. Still, they are informative for two reasons. First, they show that anthropoid primates are capable of correlating cause and effect, regardless of whether an understanding of causality has an impact on their evolutionary fitness in the wild. Second, they show that in novel contexts, primates are able to learn through unguided experimentation or emulation (see Henrich and Tennie, this volume; Rosati, this volume).

A higher cognitive requirement for using tools is a technical understanding of the objects and interactions involved in the task to be performed and, based on this understanding, selecting an appropriate tool for the task (Mulcahy and Call 2006). In most instances of primate tool use described above, appropriate tools were selected for given tasks, presumably based on the individual's ability to discriminate good from poor tools. Oftentimes, this entails an understanding of the physics of the tools and tasks. For example, the primates that habitually crack open nuts or mollusk shells must know, perhaps from experience or learning from others, that the tools they use must have certain physical properties (e.g., hardness, weight) that make them suitable for cracking open hard objects (Visalberghi et al. 2007). Thus, they select stones or large-diameter sticks rather than twigs and leaves for this task. Similarly, the use of thin probes or wands for insect fishing in chimpanzees, and at least in one instance in gorillas (Kinani and Zimmerman 2015), 
indicates that they understand that only tools of a certain diameter will fit in the termite nest's holes, and/or that using alternative tools such as clubs to gain access to the termite mound will have undesirable consequences.

Similarly, following the selection of an appropriate tool, primates must have some understanding of the physics of the interactions between tool and substrate, especially in the case of kinematics of percussive tool use. Adjusting the force required to break open a nut, for example, may not only improve the efficiency of the extractive task (i.e., optimize it), but can also minimize the risk of injury. A recent kinematic study in wild capuchins suggests that they adjust the magnitude and frequency of strikes necessary to open nuts with shells of varying hardness, even though they may use the same stone in each case (Mangalam et al. 2016). Similar observations have been made in bonobos (Neufuss et al. 2017) and chimpanzees (Boesch and Boesch 1983).

Associative tool use is cognitively more complex. Shumaker et al. (2011: 19) define associative tool use as "tools used in any combination to achieve an outcome." This includes tools that are used one after the other (sequential tools), tools that are used to acquire other tools, or combinations of tools, usually with different functions, that are used simultaneously to achieve a single outcome (composite tools; Hayashi 2015). Wild capuchins have been observed to use tools sequentially, such as stones used to pound on cavities in tree trunks, followed by the use of sticks as probes to access insect nests on the inside (Mannu and Ottoni 2009). One of the best examples of composite tool use comes from observations of nut cracking by Bossou chimpanzees (Sakura and Matsuzawa 1991; Carvalho et al. 2008, 2009). The composite tools here are the hammerstone and a movable anvil, in contrast to other sites such as Taï, where the anvil is typically an unmovable rocky outcrop. This composite tool can be even more complex, as some chimpanzees at Bossou have been observed to prop and stabilize the anvil with additional stones, leading to a final composite tool made up of four stones.

Tool manufacture also requires more advanced cognitive skills. Whereas many forms of tool behavior in primates rely on the immediate use of unmodified objects for specific tasks, tool manufacture involves a sequence of events qualitatively different from sequential or composite tool use. First, because the tool does not yet exist, a mental representation must be made of it and the use to which it will be put in some more or less distant time and place, that is, an understanding of causality in the absence of its immediate manifestation ("future planning": Rosati, this volume). A second mental 
image must also be formed that defines how the original object must be modified or manufactured into the final tool such that it will actually perform the required task.

The simplest and most frequent form of tool manufacture occurs in the context of conspecific or interspecific communication. The most common manifestation of this behavior is the use of "detached" objects, typically branches, that are then dropped from trees, thrown, or waved at others (Shumaker et al. 2011). This behavior qualifies as tool manufacture since the branch, prior to being a missile or a display, is not yet an unattached object. This behavior has been observed in the wild in many anthropoid primates, including New World monkeys (e.g., capuchins: Oppenheimer 1977), cercopithecoids (e.g., red colobus monkeys: Starin 1990), and all hominoids (gibbons: Carpenter 1940; orangutans: Galdikas 1982; gorillas: Wittiger and Sunderland-Groves 2007; bonobos: Hohmann and Fruth 2003; chimpanzees: Goodall 1986).

More complex forms of tool manufacture are associated with extractive foraging. Tools used as displays or deterrents are likely manufactured without regard to their physical properties. In other words, whatever is at hand will do to deter a potential predator or to attract the attention of a potential mate. In contrast, tools manufactured for extractive foraging must meet specific criteria. Thus, these tools require both a technical understanding and a mental template to produce and use. For example, probing an insect nest requires a twig of the right diameter, which must also be stripped of its leaves. Similarly, sticks or stones can't be used as sponges to absorb water, and hence chimpanzees deliberately strip and use leaves to soak up water from concavities (McGrew 1992). Additionally, it has been shown that chimpanzees select particular species of vegetation to make sponges for drinking water, perhaps because those raw materials are more efficient at absorbing liquid than other vegetation readily available in the same location (Tonooka 2001; Sousa 2011)

Only a few nonhuman primate taxa have been observed to manufacture tools of this kind in the wild, namely capuchin monkeys, orangutans, and chimpanzees (Goodall 1986; van Schaik et al. 1996; Mannu and Ottoni 2009). Interestingly, two manufactured tool types are common to these three species: modified sticks or twigs used for probing and collecting arthropods, and branches modified to pound, chisel, or hammer into insect nests or to open tough-skinned fruits and nuts. Additionally, wild chimpanzees have been 
observed on occasion to use stone tools that were modified in the sense that they broke during use, and one of the fragments was then used to continue the extractive foraging task, although the breakage was likely accidental (Carvalho et al. 2008).

Tool use and manufacture in the wild also involves a type of cognitive skill indirectly linked with the tool and its use, namely the ability to learn, either by trial and error or by emulation (social learning; van Schaik et al. 1999). It is unclear how much tools are spontaneously discovered or invented in the wild (but see Hobaiter et al. 2014 for a case of innovation and transmission), although simpler tools such as waving displays may arise out of a basic understanding of causality. In the context of associative tool use and tool manufacture, primatologists have observed that tool users are often in the presence of other individuals. This social influence, which also requires tolerance and gregariousness on the part of the tool user, is most frequently observed in orangutans and especially chimpanzees (Tomasello et al. 1987; Nagell et al. 1993; Call and Tomasello 1994; see also Henrich and Tennie, this volume, for a detailed discussion of teaching and social learning in chimpanzees).

The comparative evidence thus indicates that chimpanzees, and to a lesser extent orangutans and capuchins, have all the cognitive skills associated with complex tool use (causality, technical understanding, associative tool use), and also with tool manufacture (mental representation or projection). These skills are acquired in humans at a very young age (Nagell et al. 1993). Chimpanzees also have the social context that facilitates the learning and cultural transmission of tool use / manufacture skills, as do humans. Captive studies indicate that most anthropoid primates have at least the basic cognitive skills to use tools as well, suggesting that the capacity to use tools is primitive for anthropoids. Unless the more advanced cognitive skills associated with tool manufacture found in Pan and Homo evolved convergently, as likely happened between hominids and capuchins (Cebus/Sapajus), then the most parsimonious explanation is that the LCA should have had advanced technical-cognitive skills as well.

\section{Fossil and Archaeological Evidence}

Fossil primates, especially early hominins, can provide important details on the evolution of morphological correlates of tool use/manufacture, espe- 
cially hand morphology. In addition, paleoenvironmental reconstructions for fossil taxa provide important clues as to the habitats they lived in, and whether they favored tool innovation. Finally, the archaeological record itself provides indisputable proof of tool use, and especially manufacture. How complex the tools are, and when they first appear, can provide insights into the likelihood that the LCA used tools, and what kind these were.

\section{Miocene Apes}

Numerous species of Miocene ape have been described (reviewed in Begun 2010). Among these, a few taxa from different geological ages stand out because hand remains have been recovered, allowing for reconstructions of features relevant to the grips involved in locomotion versus dexterous manipulation. In a review of available hand remains from Miocene apes, Almecija and colleagues (2012) conclude that overall, these primates had hand proportions with long, highly curved finger bones and a relative thumb length intermediate between extant apes and modern humans (Almecija et al. 2012; see also Almecija et al. 2007). The structure of the thumb skeleton shows that its proximal phalanx was also highly curved, while its distal phalanx was elongate and possessed strong flexor attachments, but no apical tufts that would have supported a distal pulp (Almecija et al. 2012). An analysis of the distal thumb phalanx of the late Miocene putative hominin Orrorin tugenensis ( 6 million years ago [Ma]) by the same authors suggests a morphology that is more similar to modern humans than to extant and other Miocene apes, with distinct apical tufts, and an ungual fossa and elevated ridge marking the insertion of the flexor pollicis longus (Gommery and Senut 2006; Almecija et al. 2010).

From this fossil evidence, Almecija and colleagues infer that the primitive condition for Middle and Late Miocene apes was distinct from extant apes, with long curved fingers but a relatively long thumb, which moreover in Orrorin possessed all the markings of a thumb capable of producing strong precision grips. They conclude that the skeletal morphology of the hand in the LCA would have been more primitive than is generally assumed, with only subtle changes in proportions and thumb anatomy necessary to produce the human condition, and more marked and convergent elongation of the non-pollical digits in the extant apes (Almecija et al. 2015) (but see above, and Pilbeam and Lieberman, this volume, for an alternative interpretation of hand evolution in gorillas based on the scaling of skeletal dimensions to 
body size in the context of terrestrial locomotion). If true, this suggests that the evolution of hand proportions in the hominins was not necessarily the result of selection for increased manual dexterity (Alba et al. 2003; Rolian et al. 2010).

Paleoenvironmental reconstructions for the Middle Miocene apes most often point to seasonal, subtropical forest habitats (reviewed in Elton 2008). The inference made from these reconstructions, supported to some extent by craniodental remains, is that the Middle Miocene apes relied on the exploitation of arboreal resources as a significant portion of their diets. In contrast, paleoecological reconstructions for Late Miocene putative stem hominins such as Sahelanthropus tchadensis and Orrorin tugenensis reflect a mix of habitats including closed-canopy forests but also open woodlands and open water environments (Elton 2008). Such mixed habitats may have spurred technological innovation as new food sources became increasingly available. There is no archaeological record from this time period.

\section{Ardipithecus ramidus}

Ar. ramidus is a remarkably complete fossil dated to 4.4 Ma recovered from the Afar region of Ethiopia (White et al. 2009). The fossil remains of a female individual include the skull, a virtually complete forelimb, pelvis, and many elements of the hind limb (Suwa et al. 2009a, 2009b; Lovejoy et al. 2009a, $2009 \mathrm{~b}, 2009 \mathrm{~d})$. These remains have enabled a detailed reconstruction of its paleobiology, although some details are still a matter of debate (Lovejoy 2009a, 2009b, 2009c; Stanford 2012; Young et al. 2015). Ar. ramidus's hand remains indicate digit proportions more similar to gorillas than to either modern Pan or Homo. It also possessed relatively large joint surfaces in the wrist, a relatively long and robust thumb with a marked gable on its distal phalanx that reflects the insertion of a flexor pollicis longus (Lovejoy et al. 2009b). Lovejoy and colleagues have suggested that the hand of Ar. ramidus was capable of strong palmar grips with good opposability afforded by a more flexible wrist. Similar to Almecija et al. (2015), they conclude that the primitive condition of the LCA hand was more similar to humans than to modern African apes, although, as discussed above, and in Pilbeam and Lieberman (this volume), these conclusions may depend on the choice of body size proxies for interspecific comparisons. 
Paleoenvironmental reconstructions suggest that Ar. ramidus lived in a mixed woodland habitat with small forest patches and access to water resources, rather than in open grasslands (Louchart et al. 2009; Suwa et al. 2009b; White et al. 2009). Based on its dentition, Suwa and colleagues suggest that Ardipithecus was an omnivore, feeding in both arboreal and terrestrial environments, but showing neither the dental features associated with $\mathrm{a} \mathrm{C}_{3}$ diet, based on ripe fruit frugivory (chimpanzees), nor those associated with a more $\mathrm{C}_{4}$ diet consisting of more abrasive foods found in open grassland/savanna environments.

\section{Australopithecines}

The australopithecines are a morphologically diverse group of hominins that lived between 4.2 and 2 Ma in both East and South Africa. Australopithecines were committed bipeds, though postcranial remains are also indicative of climbing and arboreal behaviors (e.g., climbing for food resources, escape from predators, or nesting) (Berger et al. 2011; Ward et al. 2011). Hand fossils of one of the earliest members of the genus, $A u$. afarensis, indicate digit proportions that are at the lower end of the human range, overlapping with gorillas, although this is based on reconstructions from unassociated hand remains from multiple sites in the Afar region of Ethiopia (Marzke 1983; Alba et al. 2003; Rolian and Gordon 2013). The thumb metacarpal is relatively gracile has weakly developed apical tufts, but shows a clear insertion for the flexor pollicis longus (Bush et al. 1982; Ward et al. 2012). Additional hand fossils from South African members of the genus also evince hands that were modern in appearance, possibly as early as 3.7 Ma (Au. africanus: Clarke 1999), and certainly by 1.9 Ma (Au. sediba: Kivell et al. 2011).

Reconstructions of the paleoenvironmental context of australopithecines point to habitats that were cooler and more similar to modern-day woodlands and grasslands than to closed-canopy forests associated with earlier hominins (Bobe et al. 2002). The environmental shift to more open grasslands with more fragmented forest patches is often seen as being partly responsible for increased terrestriality and hence the emergence of committed bipedalism in these hominins (Dominguez-Rodrigo 2014). It is also thought to have opened up opportunities for extractive foraging of new food resources, including tubers and perhaps even scavenged meat. In other words, it may 
have provided an impetus for technological innovation in order to gain access to these new resources.

Two recent archaeological discoveries suggest $A u$. afarensis did in fact use and make stone tools. In Dikika, a 3.39 Ma site in Ethiopia, bones bearing cutmarks as well as percussive marks have been interpreted by their discoverers as indicating the use of sharp-edged stone tools for removing flesh from the bones and smashing them to access the marrow, respectively (McPherron et al. 2010; but see Dominguez-Rodrigo et al. 2010). No stone tools have been recovered from that site, though a nearby site in the same formation has yielded hominin fossils, including a juvenile attributed to $A u$. afarensis, suggesting this species may have been the maker/ user of these tools (Alemseged et al. 2006). In 2015, Harmand and colleagues described over one hundred lithic artifacts recovered from Lomekwi, Kenya, dated to 3.3 Ma. These artifacts were recovered in situ, and in the same spatiotemporal context as hominins (as yet undescribed). The assemblage is distinctive from any Oldowan collection, and includes a diversity of large artifacts, such as sharp-edged flakes, hammerstones, and potential anvils, all pointing to the significance of percussive activities in these Pliocene hominins (Harmand et al. 2015; Lewis and Harmand 2016) (Figure 17.5).

\section{Early Homo}

Our own genus first appears around 2.8 Ma (Villmoare et al. 2015). Early putative members of the genus, such as Homo habilis, had postcranial proportions more similar to Australopithecus than to later Homo, a slightly larger cranial capacity, and smaller teeth than geologically earlier australopithecines. A partial hand, $\mathrm{OH}_{7}$, recovered from Olduvai Gorge in Tanzania, was initially attributed to Homo habilis, although it may have belonged to a robust australopithecine (Moyà-Solà et al. 2008). The $\mathrm{OH} 7$ hand does not permit reconstructions of digit proportions, but possesses a distal thumb phalanx with none of the derived features of Homo hands (Almecija et al. 2010). The types of grips $H$. habilis could have generated are thus hard to reconstruct.

Regardless of the paucity of its hand remains, the first evidence of stone tool use fitting the Oldowan techno-complex is usually associated with $H$. habilis, though the 2.6 Ma assemblage from Gona, Ethiopia (Semaw et al. 1997), has been suggested to be a product of Australopithecus garhi, and multiple species living at the time were likely using stone tools, in- 


\section{A}
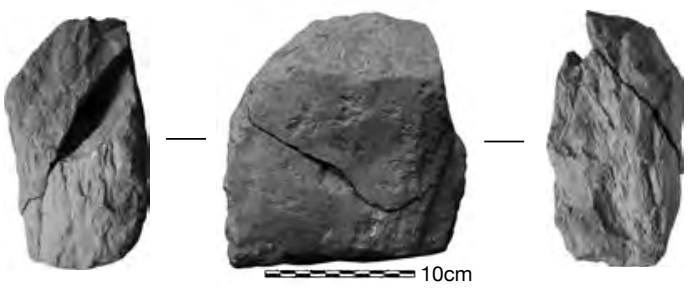

B

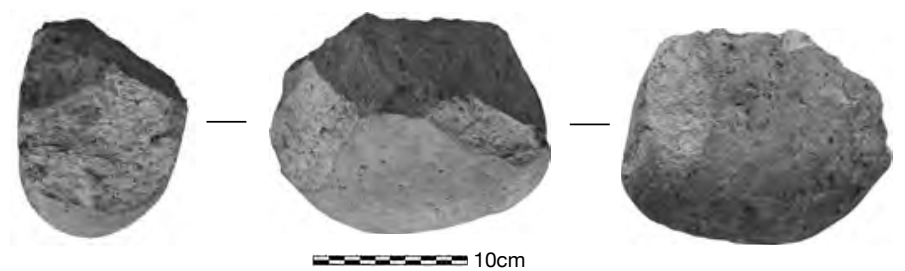

C

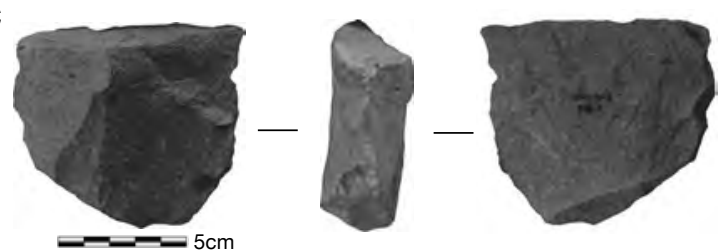

FIGURE 17.5. The Lomekwi stone tool assemblage, dated to 3.3 MYA. (a) In situ core $(1.85 \mathrm{~kg})$ and refitting surface flake $(650 \mathrm{~g})$, both displaying dispersed percussion marks on cortex. (b) In situ unifacial core. (c) Flakes showing scars of previous removals. Adapted by permission from Macmillan Publishers Ltd.: Nature (Harmand et al. 2015. 3.3-million-year-old stone tools from Lomekwi 3, West Turkana, Kenya. Nature 521: 310-315), Copyright 2015.

cluding robust australopithecines (e.g., Susman 1994). The H. habilis taxon name ("handy man") comes from its discovery in Olduvai Gorge in association with modified stone artifacts (Leakey et al. 1964). This discovery led to the description of the first lithic industry, known as the Oldowan. The Oldowan stone tool kit contains deliberately modified stone artifacts such as choppers and sharp-edged flakes, along with hammerstones involved in the manufacture of the former, most likely through one-handed percussion. The tools are assumed to have been involved in butchering activities.

All later members of the genus Homo, beginning around $2 \mathrm{Ma}$ and up to the present day, are systematically associated with lithic technology, with the 
exception of the recently described Homo naledi from South Africa (Berger et al. 2015). The lithic tool kits of hominins expands and becomes increasingly sophisticated throughout the Pleistocene, shifting away from simple but highly variable Oldowan flakes, choppers, and hammerstones, to tools that required more systematic and lengthier reduction processes (Toth and Schick 2009). This systematic processing leads to highly reproducible and uniform tools, such as the Acheulean bifaces from 1.6 Ma (Whiten et al. 2009). Such tools presumably required, at a minimum, the cognitive skill to produce a mental template of the final product and proceed through the complex series of steps that would produce it (but see Moore and Perston 2016 for an alternative view).

\section{Synthesizing the Evidence}

Did the last common ancestor of Homo and Pan use and / or make tools? The sum of the evidence described above suggests that it could. The comparative evidence from extant primates suggests that tool use has been reported in all great apes, albeit at very different frequencies, and that tool manufacture is well developed in chimpanzees and orangutans. Furthermore, anatomical evidence from the hand in extant primates suggests that a derived humanlike hand is not a prerequisite for the efficient use of tools. The fossil evidence from Miocene apes suggests that the LCA hand may actually have been more human-like than previously thought, meaning that it may have been able to generate the forceful precision grips associated with stone tool use and manufacture. Finally, the evidence indicates that most primates possess the cognitive requirements to use tools. Anthropoid primates show the cognitive skills necessary to make simple tools used for displays. Wild chimpanzees show the greatest cognitive flexibility to not only use and make tools, but to make composite tools that are task-specific and require multiple sequential steps to produce. The tolerance toward conspecifics, allowing youngsters to observe closely technological behaviors, combined with long periods of trial and error, normally within a social context, could also help to explain the emergence of more complex tool behaviors and contribute to its endurance across generations.

Thus, chimpanzees share with humans many of the anatomical, cognitive, behavioral, and even social attributes related to tool use and manufac- 
ture (Henrich and Tennie, this volume; Rosati, this volume). Based purely on principles of parsimony, then, one can conclude that the LCA had the capacity to use and make tools (Panger et al. 2002; Duda and Zrzavy 2013). However, one must also consider the possibility that tool behavior in Pan and Homo evolved convergently. After all, tool use in the wild is relatively rare among primates, and the three nonhuman primates that are thought to use and make tools for extractive foraging purposes more frequently-Pan, Pongo, and Sapajus (Cebus)-are not sister taxa.

The striking similarity in the tool behaviors of wild chimpanzees and capuchins further indicates that a more elaborate tool kit, including the use of stone tools, and the manufacture of dipping sticks and pounding sticks to extract invertebrate food resources, has evolved in parallel at least once among anthropoids (Visalberghi et al. 2015). It is important to note, however, that whereas complex and habitual tool use / manufacture appears to be universal among wild chimpanzees, it is not present among all species in the genus Sapajus, nor even in the one that has been observed most frequently to engage in this behavior (Sapajus libidinosus: Ottoni and Izar 2008). Its evolution in Sapajus groups may be an isolated event spurred on by local ecological conditions in specific populations (see below).

Whether one believes that the LCA had the capacity to use and make tools ultimately boils down to how much homoplasy one is ready to accept among sister taxa. Complex tool behavior, including variability in cultural traditions across groups and habitual tool manufacture, is universal among chimpanzees and humans, and has now been documented in a semi-wild context for bonobos (Neufuss et al. 2017). As such, we find it unlikely that such an event would have evolved in parallel between sister taxa, to the extent that it became fixed as an autapomorphy among all populations in both lineages. Simply put, we believe the last common ancestor of Pan and Homo could use tools.

Yet arguing that the LCA could use tools is not the same as stating that it did. In other words, the primitive condition in the LCA was not necessarily the existence of complex tool culture and behavior prior to the divergence of Pan and hominins, but rather the presence of the necessary features to develop it under the right circumstances. In fact, looking across anthropoid primates, the comparative behavioral and cognitive evidence discussed in this chapter, in captivity and in the wild, suggests that the potential for tool use/manufacture may be the primitive condition for all anthropoid primates. If true, 
then this suggests that the absence of tool behaviors among most wild primates is not due to a cognitive or manipulative inability to use, or even make, tools, but rather because the circumstances that favor technological behaviors and innovation are absent for most species. If the propensity for technological behavior is a primitive condition in higher primates, then this may also explain the convergent nature of tool behavior among distantly related primates: all higher primates have the potential use tools, but the impetus to do so is context-dependent.

So what exactly are the contexts that are conducive to realizing tool using/making potential in primates? One of the most important conditions for the presence or development of tool use in primates may be the availability and likelihood of finding food resources that require extractive processing. These foods, such as shelled nuts, nest-dwelling arthropods, tubers, roots, and mollusks, have high nutritional payoffs, but require extraction beyond the capabilities of hands or teeth. As in captive experiments, they present a problem that must be solved, and only the most innovative individuals capable of leveraging their immediate environment will gain access to the food. The opportunity for innovation is thus a key prerequisite, and a predictor, of tool use and manufacture (van Schaik et al. 1999; Koops et al. 2015).

In this context, it is not a coincidence that primate species that use tools most frequently are exploiting resources with high nutritional payoffs, but high investments in terms of extractive processing. Conversely, many primates that show no evidence of tool use/manufacture in the wild may simply lack the opportunity or motivation to do so. Lack of opportunity may stem from the absence of such high-reward food items in their proximate environment. More likely, the lack of motivation may be because these primates, especially frugivores and folivores, can "get by" on diets consisting of foods with lower nutritional value but equally low investment, and there is little reproductive advantage in accessing other, higher-payoff foods.

Some habitats may be more conducive to tool use/manufacture than others, for example, because of the availability of food sources that create opportunities for innovation and social learning. Meulman and colleagues (Meulman et al. 2012) have argued that partially terrestrial environments play an important role in the development of complex tool technology in primates, especially lithic technology. They cite four lines of evidence to support this view. First, the capuchin monkeys most often observed using stone tools in the wild live in savanna-like environments, while more arboreal pop- 
ulations of the same taxon do not exhibit this behavior (Visalberghi and Fragaszy 2013). Second, chimpanzees, which are more terrestrial than orangutans, show more complex tool variants than the latter (the authors define complex tools as those that are acquired in part through social learning, are used flexibly, and can be accumulated). Third, among chimpanzees, tools used in terrestrial contexts are more complex (e.g., composite or sequential) than those used in exclusively arboreal environments. The authors ascribe this difference to availability of extractive foraging opportunities, to the opportunity to use their hands without the constraints of an arboreal setting, and to the greater opportunities for social learning in terrestrial settings. Finally, they suggest that the greater frequency of tool behaviors observed in captivity may be due to a "terrestriality effect," in which even the most arboreal primates spend more time on the ground than they would in the wild.

Thus, knowing whether the LCA actually used and made tools may greatly depend on reconstructions of its paleoenvironment, and especially whether this environment facilitated terrestrial behaviors (Elton 2008). As there are no fossils from the LCA, it is challenging to reconstruct its exact paleoenvironment. Nonetheless, if one accepts that it lived in Africa between 6 and $9 \mathrm{Ma}$ (Moorjani et al. 2016), then indirect evidence of its habitats may be gleaned from Late Miocene fossil/geological sites in Africa. The Late Miocene record in Africa is spotty at best (reviewed in Elton 2008), and in fact many of our insights into the LCA's habitat come from paleoenvironmental reconstructions of the earliest putative hominins, Sahelanthropus tchadensis ( $7 \mathrm{Ma}$ : Vignaud et al. 2002), Orrorin tugenensis ( 6 Ma: Pickford and Senut 2001) and Ardipithecus kadabba ( 5.2-5.8 Ma: WoldeGabriel et al. 2001).

Habitat reconstructions for these putative stem hominins are fraught with difficulties, but the general pattern that emerges is one of mosaic environments, the product of increasing climatic variability. Mosaic habitats, as the name implies, include "a range of different habitat types, scattered across and interspersed within a given area" (Elton 2008: 381). While this may not be a satisfactory way of reconstructing the Late Miocene environment of the LCA, it does suggest that its habitat was neither closed tropical forest, nor open grassland/savanna. It was more likely woodland with interrupted canopy forests, greater seasonality, and perhaps access to more open spaces, which may have required some degree of terrestriality, all the while preserving an important arboreal component in the LCA behavioral repertoire. Some degree of bipedalism has also been inferred for these Late Miocene stem 
hominins, and for Ardipithecus ramidus, reinforcing the notion that they and the LCA may have been semi-terrestrial.

If, as the evidence suggests, the LCA lived in a mosaic environment with increasing opportunities for terrestriality and access to food resources associated with terrestrial environments (e.g., roots, tubers, termite mounds), and filled all the prerequisites for tool use / manufacture, then it is highly likely that it did use and make tools. Specifically, its tool kit was probably most similar to the tool kit of chimpanzee populations that live in mosaic habitats, subject to higher ecological variability, with more seasonal environments in which availability of arboreal versus terrestrial foods varies due to fluctuations in temperature and / or rainfall, such as Bossou in West Africa (Yamakoshi 1998; Hockings et al. 2015).

In concrete terms, its tool kit likely included organic tools that were used in extractive foraging, grooming, and display activities, such as leaf sponges, dipping sticks, and modified branches. Some of these tools need to be deliberately modified, implying tool manufacture in the LCA as well. Whether it used stone tools is an open question (Whiten 2011; Haslam 2014). Whereas many organic tools and associated behaviors are shared across African chimpanzee populations and bonobos (e.g., branch shaking and leaves as tools), the use of stone tools for pounding and opening nuts, and nut cracking itself, is best known in West African chimpanzees (Whiten et al. 1999; Carvalho et al. 2008), although recent evidence indicates that semiwild (i.e., rehabilitated) bonobos also partake in these activities (Neufuss et al. 2017). Outside of hominins, stone tool use for pounding appears to have evolved independently in Pan troglodytes, Sapajus libidinosus, and Macaca fascicularis aurea. Because it is not customary among all chimpanzee subspecies or populations, and because it is exceedingly rare in bonobos, Haslam (2014) argues that stone tool use was unlikely in the ancestor of bonobos and chimpanzees. If this is true, then the most parsimonious scenario is that the Pan-Homo last common ancestor also did not use stone tools. However, the absence of stone tool use in chimpanzees in East Africa, and in bonobos, could equally be due to lack of ecological opportunity to innovate, develop, and maintain stone tool behaviors, and the most recent evidence (Harmand et al. 2015) reinforces the idea that stone tool use is shared by a larger number of hominin genera (Australopithecus, Kenyanthropus, Homo), as well as by modern Pan and Homo. If the LCA did use stone tools, however, we expect based on comparative evidence from extant pri- 
mates that these assemblages would have been scarce, with relatively low numbers of artifacts per site, and largely composed of unmodified hammers, and possibly anvils, used in percussive activities.

\section{Conclusion}

We may never recover fossil or archaeological evidence of the LCA, and thus know with certainty that it used or made tools. However, given the shared complexity of tool use and manufacture in wild chimpanzees and hominins, which is both qualitatively and quantitatively different from other primates, the most parsimonious scenario, at least from a phylogenetic perspective, is that the LCA did use and make tools. Even without invoking parsimony, the comparative anatomical, cognitive, fossil, and archaeological evidence discussed in this chapter strongly suggests that the LCA had the capacity to and did use and manufacture tools. These tools were probably mostly organic, used for displays, grooming, and especially extractive foraging, but also likely included a small lithic component. In the absence of "smoking gun" fossil and archaeological evidence, future comparative studies on the habitat and behavioral ecology of extant primates in the wild (e.g., terrestriality), and on paleoenvironmental reconstructions of the LCA's habitat during the Late Miocene of Africa, will continue to refine our understanding of the tool types and behaviors of the LCA.

\section{Summary}

Although often still considered a hallmark of humans and their ancestors, the use and manufacture of tools for hygiene, communication, and extractive foraging is now well documented in other primates, including capuchins, macaques, and most hominoids. A so-called tool culture is particularly well documented in chimpanzees, and present in bonobos. Consequently, the comparative study of the behavioral variation in tool repertoires, but also of the morphological and cognitive prerequisites for tool use in extant Homo and Pan, can be used to make inferences about the tools and tool-making capabilities of the LCA. Comparative tool use in nonhuman primates, comparative anatomy and biomechanics of primate hands, comparative cognition 
in hominoids, and evidence from the fossil and archaeological records of early hominins, all support the idea that the LCA was capable of using and manufacturing stone tools for percussive activities (e.g., cracking nuts), and likely also organic tools (e.g., modified sticks for extractive foraging or displays). Whether the LCA actually used or made tools was likely contingent on whether its environment provided enough opportunities to encounter and exploit key resources. Specifically, the distribution of food sources and raw material availability and quality would have been important factors determining opportunities for innovation and for transmission and diffusion of technological behaviors.

\section{Acknowledgments}

We thank Martin Muller, David Pilbeam, and Richard Wrangham for inviting us to contribute to this volume, as well as for providing helpful feedback on earlier versions of the manuscript.

\section{References}

Alba, D. M., S. Moyà-Solà, and M. Kohler. 2003. Morphological affinities of the Australopithecus afarensis hand on the basis of manual proportions and relative thumb length. Journal of Human Evolution 44: 225-254.

Alemseged, Z., F. Spoor, W. H. Kimbel, R. Bobe, D. Geraads, D. Reed, and J. G. Wynn. 2006. A juvenile early hominin skeleton from Dikika, Ethiopia. Nature 443: 296-301.

Almecija, S., D. M. Alba, and S. Moyà-Solà. 2012. The thumb of Miocene apes: New insights from Castell de Barbera (Catalonia, Spain). American Journal of Physical Anthropology 148; 436-450.

Almecija, S., D. M. Alba, S. Moyà-Solà, and M. Kohler. 2007. Orang-like manual adaptations in the fossil hominoid Hispanopithecus laietanus: First steps towards great ape suspensory behaviours. Proceedings of the Royal Society B: Biological Sciences 274: 2375-2384.

Almecija, S., S. Moyà-Solà, and D. M. Alba. 2010. Early origin for human-like precision grasping: A comparative study of pollical distal phalanges in fossil hominins. PLOS ONE 5: e11727.

Almecija, S., J. B. Smaers, and W. L. Jungers. 2015. The evolution of human and ape hand proportions. Nature Communications 6: 7717. 
Badrian, N. 1981. Preliminary observations on the feeding behavior of Pan paniscus in the Lomako forest of central Zaire. Primates 22: 173-181.

Begun, D. R. 2010. Miocene hominids and the origins of the African apes and humans. Annual Review of Anthropology 39: 67-84.

Berger, L. R., J. Hawks, D. J. de Ruiter, S. E. Churchill, P. Schmid, L. K. Delezene, T. L. Kivell, H. M. Garvin, S. A. Williams, J. M. DeSilva, M. M. Skinner, C. M. Musiba, N. Cameron, T. W. Holliday, W. Harcourt-Smith, R. R. Ackermann, M. Bastir, B. Bogin, D. Bolter, J. Brophy, Z. D. Cofran, K. A. Congdon, A. S. Deane, M. Dembo, M. Drapeau, M. C. Elliott, E. M. Feuerriegel, D. Garcia-Martinez, D. J. Green, A. Gurtov, J. D. Irish, A. Kruger, M. F. Laird, D. Marchi, M. R. Meyer, S. Nalla, E. W. Negash, C. M. Orr, D. Radovcic, L. Schroeder, J. E. Scott, Z. Throckmorton, M. W. Tocheri, C. VanSickle, C. S. Walker, P. P. Wei, and B. Zipfel. 2015. Homo naledi, a new species of the genus Homo from the Dinaledi Chamber, South Africa. Elife 4: eog56o.

Berger, L. R., J. Kibii, S. Churchill, P. Schmid, K. Carlson, B. de Klerk, D. de Ruiter, T. Holliday, T. Kivell, J. Gurche, B. Zipfel, J. DeSilva, and R. Kidd. 2011. New remains of Australopithecus sediba from the Malapa site, South Africa. American Journal of Physical Anthropology 144: 88-88.

Biro, D., N. Inoue-Nakamura, R. Tonooka, G. Yamakoshi, C. Sousa, and T. Matsuzawa. 2003. Cultural innovation and transmission of tool use in wild chimpanzees: evidence from field experiments. Animal Cognition 6: 213-223.

Biro, D., C. Sousa, and T. Matsuzawa. 2006. Ontogeny and cultural propagation of tool use by wild chimpanzees at Bossou, Guinea: Case studies in nut cracking and leaf folding. In T. Matsuzawa, M. Tomonaga, and M. Tanaka, eds., Cognitive Development in Chimpanzees, 476-508. Tokyo: Springer Tokyo.

Bobe, R., A. K. Behrensmeyer, and R. E. Chapman. 2002. Faunal change, environmental variability and late Pliocene hominin evolution. Journal of Human Evolution 42: 475-497.

Boesch, C., and H. Boesch. 1983. Optimization of nut-cracking with natural hammers by wild chimpanzees. Behaviour 83: 265-286.

Boesch, C., and H. Boesch. 1993. Different hand postures for pounding nuts with natural hammers by wild chimpanzees. In H. Preuschoft and D. J. Chivers, eds.), Hands of Primates, 31-43. Vienna: Springer.

Boesch, C., and H. Boesch-Achermann. 200o. The Chimpanzees of the Taï Forest: Behavioural Ecology and Evolution. Oxford: Oxford University Press.

Boesch, C., J. Head, and M. M. Robbins. 2009. Complex tool sets for honey extraction among chimpanzees in Loango National Park, Gabon. Journal of Human Evolution 56: 560-569.

Breuer, T., M. Ndoundou-Hockemba, and V. Fishlock. 2005. First observation of tool use in wild gorillas. PLoS Biology 3: 2041-2043.

Bush, M. E., C. O. Lovejoy, D. C. Johanson, and Y. Coppens. 1982. Hominid carpal, metacarpal, and phalangeal bones recovered from the Hadar formation: 1974-1977 collections. American Journal of Physical Anthropology 57: 651-677. 
Call, J., and M. Tomasello. 1994. Production and comprehension of referential pointing by orangutans (Pongo pygmaeus). Journal of Comparative Psychology 108: 307-317.

Carpenter, C. R. 1940. A Field Study in Siam of the Behavior and Social Relations of the Gibbon (Hylobates lar). Baltimore: Johns Hopkins Press.

Carvalho, S., D. Biro, W. C. McGrew, and T. Matsuzawa. 2009. Tool-composite reuse in wild chimpanzees (Pan troglodytes): Archaeologically invisible steps in the technological evolution of early hominins? Animal Cognition 12: S103-S114.

Carvalho, S., E. Cunha, C. Sousa, and T. Matsuzawa. 2008. Chaînes opératoires and resource-exploitation strategies in chimpanzee (Pan troglodytes) nut cracking. Journal of Human Evolution 55: 148-163.

Carvalho, S., and W. McGrew. 2012. The origins of the Oldowan: Why chimpanzees (Pan troglodytes) still are good models for technological evolution in Africa. In M. Domínguez-Rodrigo, ed., Stone Tools and Fossil Bones: Debates in the Archaeology of Human Origins, 201-221. Cambridge: Cambridge University Press.

Chiang, M. 1967. Use of tools by wild macaque monkeys in Singapore. Nature 214: 1258-1259.

Clarke, R. J. 1999. Discovery of complete arm and hand of the 3.3 million-year-old Australopithecus skeleton from Sterkfontein. South African Journal of Science 95: 477-480.

Darwin, C. 1871. The Descent of Man, and Selection in Relation to Sex. New York: D. Appleton.

Deng, H., and J. Zhou. 2016. "Juggling" behavior in wild Hainan gibbons, a new finding in nonhuman primates. Scientific Reports 6: 23566.

Dominguez-Rodrigo, M. 2014. Is the "Savanna Hypothesis" a dead concept for explaining the emergence of the earliest hominins? Current Anthropology 55: 59-81.

Dominguez-Rodrigo, M., A. Z. P. Mabulla, H. T. Bunn, F. Diez-Martin, E. Baquedano, D. Barboni, R. Barba, S. Dominguez-Solera, P. Sanchez, G. M. Ashley, and J. Yravedra. 2010. Disentangling hominin and carnivore activities near a spring at FLK North (Olduvai Gorge, Tanzania). Quaternary Research 74: 363-375.

Duda, P., and J. Zrzavy. 2013. Evolution of life history and behavior in Hominidae: Towards phylogenetic reconstruction of the chimpanzee-human last common ancestor. Journal of Human Evolution 65: 424-446.

Elton, S. 2008. The environmental context of human evolutionary history in Eurasia and Africa. Journal of Anatomy $212: 377-393$.

Fan, P. F., Q. Y. Ni, G. Z. Sun, B. Huang, and X. L. Jiang. 2009. Gibbons under seasonal stress: The diet of the black crested gibbon (Nomascus concolor) on Mt. Wuliang, central Yunnan, China. Primates 50: 37-44.

Fushimi, T., O. Sakura, T. Matsuzawa, H. Ohno, and Y. Sugiyama. 1991. Nut-cracking behavior of wild chimpanzees (Pan troglodytes) in Bossou, Guinea (West Africa). In A. Ehara, T. Kimura, O. Takenaka, and M. Iwamoto, eds., Primatology Today, 695-696. Amsterdam: Elsevier. 
Galdikas, B. M. F. 1982. Orangutan tool-use at Tanjung-Puting-Reserve, central Indonesian Borneo (Kalimantan-Tengah). Journal of Human Evolution 11: 19-33.

Gommery, D., and B. Senut. 2006. The terminal thumb phalanx of Orrorin tugenensis (Upper Miocene of Kenya). Geobios-Lyon 39: 372-384.

Goodall, J. 1963. My life among wild chimpanzees. National Geographic 124: 273-308.

Goodall, J. 1986. The Chimpanzees of Gombe: Patterns of Behavior. Cambridge, MA: Harvard University Press.

Gruber, T., and Z. Clay. 2016. A comparison between bonobos and chimpanzees: A review and update. Evolutionary Anthropology 25: 239-252.

Gumert, M. D., M. Kluck, and S. Malaivijitnond. 2009. The physical characteristics and usage patterns of stone axe and pounding hammers used by long-tailed macaques in the Andaman Sea region of Thailand. American Journal of Primatology 71: 594-608.

Hamilton, W. J., R. E. Buskirk, and W. H. Buskirk. 1975. Chacma baboon tactics during intertroop encounters. Journal of Mammalogy 56: 857-870.

Harmand, S., J. E. Lewis, C. S. Feibel, C. J. Lepre, S. Prat, A. Lenoble, X. Boes, R. L. Quinn, M. Brenet, A. Arroyo, N. Taylor, S. Clement, G. Daver, J. P. Brugal, L. Leakey, R. A. Mortlock, J. D. Wright, S. Lokorodi, C. Kirwa, D. V. Kent, and H. Roche. 2015. 3.3-million-year-old stone tools from Lomekwi 3, West Turkana, Kenya. Nature 521: 310-315.

Haslam, M. 2014. On the tool use behavior of the bonobo-chimpanzee last common ancestor, and the origins of hominine stone tool use. American Journal of Primatology 76: 910-918.

Haslam, M., L. Luncz, A. Pascual-Garrido, T. Falótico, S. Malaivijitnond, and M. Gumert. 2016a. Archaeological excavation of wild macaque stone tools. Journal of Human Evolution 96: 134-138.

Haslam, M., L. V. Luncz, R. A. Staff, F. Bradshaw, E. B. Ottoni, and T. Falótico. 2016b. Pre-Columbian monkey tools. Current Biology 26: R521-R522.

Hayashi, M. 2015. Perspectives on object manipulation and action grammar for percussive actions in primates. Philosophical Transactions of the Royal Society B: Biological Sciences 370: 20140350.

Hobaiter, C., T. Poisot, K. Zuberbuhler, W. Hoppitt, and T. Gruber. 2014. Social network analysis shows direct evidence for social transmission of tool use in wild chimpanzees. PLoS Biology 12: e1001960.

Hockings, K. J., M. R. McLennan, S. Carvalho, M. Ancrenaz, R. Bobe, R. W. Byrne, R. I. M. Dunbar, T. Matsuzawa, W. C. McGrew, E. A. Williamson, M. L. Wilson, B. Wood, R. W. Wrangham, and C. M. Hill. 2015. Apes in the Anthropocene: Flexibility and survival. Trends in Ecology and Evolution 30: 215-222.

Hohmann, G., and B. Fruth. 2003. Culture in bonobos? Between-species and withinspecies variation in behavior. Current Anthropology 44: 563-571.

Kinani, J.-F., and D. Zimmerman. 2015. Tool use for food acquisition in a wild mountain gorilla (Gorilla beringei beringei). American Journal of Primatology 77: 353-357. 
Kivell, T. L., J. M. Kibii, S. E. Churchill, P. Schmid, and L. R. Berger. 2011. Australopithecus sediba hand demonstrates mosaic evolution of locomotor and manipulative abilities. Science 333: 1411-1417.

Koops, K., T. Furuichi, and C. Hashimoto. 2015. Chimpanzees and bonobos differ in intrinsic motivation for tool use. Scientific Reports 5: 11356.

Koops, K., W. C. McGrew, and T. Matsuzawa. 2010. Do chimpanzees (Pan troglodytes) use cleavers and anvils to fracture Treculia africana fruits? Preliminary data on a new form of percussive technology. Primates 51: 175-178.

Koops, K., W. C. McGrew, and T. Matsuzawa. 2013. Ecology of culture: Do environmental factors influence foraging tool use in wild chimpanzees, Pan troglodytes verus? Animal Behaviour 85: 175-185.

Koops, K., E. Visalberghi, and C. P. van Schaik. 2014. The ecology of primate material culture. Biology Letters 10: 20140508.

Lapuente, J., T. C. Hicks, and K. E. Linsenmair. 2016. Fluid dipping technology of chimpanzees in Comoé National Park, Ivory Coast. American Journal of Primatology 79: e22628.

Leakey, L. S. B., J. R. Napier, and P. V. Tobias. 1964. New species of genus Homo from Olduvai Gorge. Nature 202: 7-9.

Lewis, J. E., and S. Harmand. 2016. An earlier origin for stone tool making: Implications for cognitive evolution and the transition to Homo. Philosophical Transactions of the Royal Society B: Biological Sciences 371: 20150233.

Linscheid, R., K. An, and M. Gross. 1991. Quantitative analysis of the intrinsic muscles of the hand. Clinical Anatomy 4: 265-284.

Liu, Q., D. Fragaszy, B. Wright, K. Wright, P. Izar, and E. Visalberghi. 2011. Wild bearded capuchin monkeys (Cebus libidinosus) place nuts in anvils selectively. Animal Behaviour 81: 297-305.

Lonsdorf, E. V., L. E. Eberly, and A. E. Pusey. 2004. Sex differences in learning in chimpanzees. Nature 428: 715-716.

Louchart, A., H. Wesselman, R. J. Blumenschine, L. J. Hlusko, J. K. Njau, M. T. Black, M. Asnake, and T. D. White. 2009. Taphonomic, avian, and small-vertebrate indicators of Ardipithecus ramidus habitat. Science 326: 66e1-4.

Lovejoy, C. O. 2009. Reexamining human origins in light of Ardipithecus ramidus. Science 326: 74e1-8.

Lovejoy, C. O., B. Latimer, G. Suwa, B. Asfaw, and T. D. White. 2009a. Combining prehension and propulsion: The foot of Ardipithecus ramidus. Science 326: 72e71-78.

Lovejoy, C. O., S. W. Simpson, T. D. White, B. Asfaw, and G. Suwa. 2009b. Careful climbing in the Miocene: The forelimbs of Ardipithecus ramidus and humans are primitive. Science 326: 70e1-8.

Lovejoy, C. O., G. Suwa, S. W. Simpson, J. H. Matternes, and T. D. White, T. D. 2009 c. The great divides: Ardipithecus ramidus reveals the postcrania of our last common ancestors with African apes. Science 326: 100-106.

Lovejoy, C. O., G. Suwa, L. Spurlock, B. Asfaw, and T. D. White. 2009d. The pelvis and femur of Ardipithecus ramidus: The emergence of upright walking. Science 326: 71e1-6. 
Malaivijitnond, S., C. Lekprayoon, N. Tandavanittj, S. Panha, C. Cheewatham, and Y. Hamada. 2007. Stone-tool usage by Thai long-tailed macaques (Macaca fascicularis). American Journal of Primatology 69: 227-233.

Mangalam, M., P. Izar, E. Visalberghi, and D. M. Fragaszy. 2016. Task-specific temporal organization of percussive movements in wild bearded capuchin monkeys. Animal Behaviour 114: 129-137.

Mannu, M., and E. B. Ottoni. 2009. The enhanced tool-kit of two groups of wild bearded capuchin monkeys in the Caatinga: Tool making, associative use, and secondary tools. American Journal of Primatology 71: 242-251.

Marzke, M. W. 1983. Joint functions and grips of the Australopithecus afarensis hand, with special reference to the region of the capitate. Journal of Human Evolution 12: 197-211.

Marzke, M. W. 1992. Evolutionary development of the human thumb. Hand Clinics 8: $1-8$.

Marzke, M. W. 1997. Precision grips, hand morphology, and tools. American Journal of Physical Anthropology 102: 91-110.

Marzke, M. W. 2013. Tool making, hand morphology and fossil hominins. Philosophical transactions of the Royal Society of London B 368: 20120414.

Marzke, M. W., and R. F. Marzke. 200o. Evolution of the human hand: Approaches to acquiring, analysing and interpreting the anatomical evidence. Journal of Anatomy 197: 121-140.

Marzke, M. W., R. F. Marzke, R. L. Linscheid, P. Smutz, B. Steinberg, S. Reece, and K. N. An. 1999. Chimpanzee thumb muscle cross sections, moment arms and potential torques, and comparisons with humans. American Journal of Physical Anthropology 110: $163-178$.

Marzke, M. W., and M. Shackley. 1986. Hominid hand use in the Pliocene and Pleistocene: Evidence from experimental archaeology and comparative morphology. Journal of Human Evolution 15: 439-460.

Marzke, M. W., K. L. Wullstein, and S. F. Viegas. 1992. Evolution of the power (squeeze) grip and its morphological correlates in hominids. American Journal of Physical Anthropology 89: 283-298.

Matsuzawa, T. 1996. Chimpanzee intelligence in nature and in captivity: Isomorphism of symbol use and tool use. In W. McGrew, L. F. Marchant, and J. Nishida, eds., Great Ape Societies. Cambridge: Cambridge University Press.

Matsuzawa, T. 1999. Communication and tool use in chimpanzees: Cultural and social contexts. In M. Hauser and M. Konishi, eds., The Design of Animal Communication, 645-671. Cambridge, MA: MIT Press.

Matsuzawa, T., D. Biro, T. Humle, N. Inoue-Nakamura, R. Tonooka, and G. Yamakoshi. 2001. Emergence of culture in wild chimpanzees: Education by masterapprenticeship. In T. Matsuzawa, ed., Primate Origins of Human Cognition and Behavior, 557-574. Tokyo: Springer Japan.

McGrew, W. C. 1974. Tool use by wild chimpanzees in feeding upon driver ants. Journal of Human Evolution 3: 501-504. 
McGrew, W. C. 1992. Chimpanzee Material Culture: Implications for Human Evolution. Cambridge: Cambridge University Press.

McGrew, W. C. 2004. The Cultured Chimpanzee: Reflections on Cultural Primatology. Cambridge: Cambridge University Press.

McGrew, W. C. 2010. In search of the last common ancestor: New findings on wild chimpanzees. Philosophical Transactions of the Royal Society of London B 365: 3267-3276.

McGrew, W. C. 2017. Field studies of Pan troglodytes reviewed and comprehensively mapped, focusing on Japan's contribution to cultural primatology. Primates 58: 237-258.

McGrew, W. C., L. F. Marchant, M. M. Beuerlein, D. Vrancken, B. Fruth, and G. Hohmann. 2007. Prospects for bonobo insectivory: Lui Kotal, Democratic Republic of Congo. International Journal of Primatology 28: 1237-1252.

McPherron, S. P., Z. Alemseged, C. W. Marean, J. G. Wynn, D. Reed, D. Geraads, R. Bobe, and H. A. Bearat. 2010. Evidence for stone-tool-assisted consumption of animal tissues before 3.39 million years ago at Dikika, Ethiopia. Nature 466: 857-860.

Meulman, E. J. M., C. M. Sanz, E. Visalberghi, and C. P. van Schaik. 2012. The role of terrestriality in promoting primate technology. Evolutionary Anthropology 21: 58-68.

Meulman, E. J. M., and C. P. van Schaik. 2013. Orangutan tool use and the evolution of technology. In C. Sanz, J. Call, and C. Boesch, eds., Tool Use in Animals: Cognition and Ecology, 176-202. Cambridge: Cambridge University Press.

Mittra, E. S., H. F. Smith, P. Lemelin, and W. L. Jungers. 2007. Comparative morphometrics of the primate apical tuft. American Journal of Physical Anthropology 134: 449-459.

Moore, M. W., and Y. Perston. 2016. Experimental insights into the cognitive significance of early stone tools. PLOS ONE 11: e0158803.

Moorjani, P., C. E. G. Amorim, P. F. Arndt, and M. Przeworski. 2016. Variation in the molecular clock of primates. Proceedings of the National Academy of Sciences 113: 10607-10612.

Moyà-Solà, S., M. Kohler, D. M. Alba, and S. Almecija. 2008. Taxonomic attribution of the Olduvai Hominid 7 manual remains and the functional interpretation of hand morphology in robust australopithecines. Folia Primatologica 79: 215-250.

Mulcahy, N. J., and J. Call. 2006. Apes save tools for future use. Science 312: 1038-1040.

Musgrave, S., D. Morgan, E. Lonsdorf, R. Mundry, and C. Sanz. 2016. Tool transfers are a form of teaching among chimpanzees. Scientific Reports 6: 34783 .

Nagell, K., R. S. Olguin, and M. Tomasello. 1993. Processes of social-learning in the tool use of chimpanzees (Pan troglodytes) and human children (Homo sapiens). Journal of Comparative Psychology 107: 174-186.

Napier, J. R. 1956. The prehensile movements of the human hand. Journal of Bone and Joint Surgery 38: 902-913.

Napier, J. R. 1980. Hands. New York: Pantheon. 
Neufuss, J., T. Humle, A. Cremaschi, and T. L. Kivell. 2017. Nut-cracking behaviour in wild-born, rehabilitated bonobos (Pan paniscus): A comprehensive study of handpreference, hand grips and efficiency. American Journal of Primatology 79: 1-16.

Oppenheimer, J. 1977. Forest structure and its relation to activity of the capuchin monkey (Cebus). In T. C. A. Kumar and M. R. N. Prasad, eds., Use of Non-Human Primates in Biomedical Research, 74-84. New Delhi: Indian National Science Academy.

Ottoni, E. B., and P. Izar. 2008. Capuchin monkey tool use: Overview and implications. Evolutionary Anthropology 17: 171-178.

Palombit, R. A. 1997. Inter- and intraspecific variation in the diets of sympatric siamang (Hylobates syndactylus) and lar gibbons (Hylobates lar). Folia Primatologica 68: 321-337.

Panger, M. A., A. S. Brooks, B. G. Richmond, and B. Wood. 2002. Older than the Oldowan? Rethinking the emergence of hominin tool use. Evolutionary Anthropology 11: $235-245$.

Pickford, M., and B. Senut. 2001. The geological and faunal context of Late Miocene hominid remains from Lukeino, Kenya. Comptes Rendus de l'Academie des Sciences, Serie II, Fascicule A: Sciences de la Terre et des Planetes 332 : 145-152.

Proffitt, T., L. V. Luncz, T. Falótico, E. B. Ottoni, I. de la Torre, and M. Haslam. 2016. Wild monkeys flake stone tools. Nature 539: 85-88.

Pruetz, J. D., and P. Bertolani. 2007. Savanna chimpanzees, Pan troglodytes verus, hunt with tools. Current Biology 17: 412-417.

Richmond, B. G. 2007. Biomechanics of phalangeal curvature. Journal of Human Evolution 53: 678-690.

Rolian, C., and A. D. Gordon. 2013. Reassessing manual proportions in Australopithecus afarensis. American Journal of Physical Anthropology 152: 393-406.

Rolian, C., D. E. Lieberman, and B. Hallgrimsson. 2010. The coevolution of human hands and feet. Evolution 64: 1558-1568.

Rolian, C., D. E. Lieberman, and J. P. Zermeno. 2011. Hand biomechanics during simulated stone tool use. Journal of Human Evolution 61: 26-41.

Sakura, O., and T. Matsuzawa. 1991. Flexibility of wild chimpanzee nut-cracking behavior using stone hammers and anvils: An experimental analysis. Ethology 87: 237-248.

Santos, L. R., N. Mahajan, and J. L. Barnes. 2005. How prosimian primates represent tools: Experiments with two lemur species (Eulemur fulvus and Lemur catta). Journal of Comparative Psychology 119: 394-403.

Sanz, C., D. Morgan, and S. Gulick. 2004. New insights into chimpanzees, tools, and termites from the Congo basin. American Naturalist 164: 567-581.

Sanz, C. M., and D. B. Morgan. 2007. Chimpanzee tool technology in the Goualougo Triangle, Republic of Congo. Journal of Human Evolution 52: 420-433.

Sanz, C. M., and D. B. Morgan. 2009. Flexible and persistent tool-using strategies in honey-gathering by wild chimpanzees. International Journal of Primatology 30 : 411-427. 
Semaw, S. P. Renne, J. W. K. Harris, C. S. Feibel, R. L. Bernor, N. Fesseha, and K. Mowbray. 1997. 2.5-million-year-old stone tools from Gona, Ethiopia. Nature 385: 333-336.

Shrewsbury, M. M., M. W. Marzke, R. L. Linscheid, and S. P. Reece. 2003. Comparative morphology of the pollical distal phalanx. American Journal of Physical Anthropology 121: 30-47.

Shumaker, R. W., K. R. Walkup, and B. B. Beck. 2011. Animal Tool Behavior: The Use and Manufacture of Tools by Animals. Baltimore: Johns Hopkins University Press.

Sousa, C. 2011. Use of leaves for drinking water. In T. Matsuzawa, T. Humle, and Y. Sugiyama, eds., The Chimpanzees of Bossou and Nimba, 85-96. Tokyo: Springer Japan.

Stanford, C. B. 2012. Chimpanzees and the behavior of Ardipithecus ramidus. Annual Review of Anthropology 41: 139-149.

Starin, E. D. 1990. Object manipulation by wild red colobus monkeys living in the Abuko Nature-Reserve, the Gambia. Primates 31: 385-391.

Susman, R. L. 1994. Fossil evidence for early hominid tool use. Science 265: 1570-1573.

Susman, R. L. 1998. Hand function and tool behavior in early hominids. Journal of Human Evolution 35: 23-46.

Suwa, G., B. Asfaw, R. T. Kono, D. Kubo, C. O. Lovejoy, and T. D. White. 2009a. The Ardipithecus ramidus skull and its implications for hominid origins. Science 326: 68e1-7.

Suwa, G., R. T. Kono, S. W. Simpson, B. Asfaw, C. O. Lovejoy, and T. D. White. 2009 b. Paleobiological implications of the Ardipithecus ramidus dentition. Science 326: 94-99.

Thorpe, S. K. S., R. H. Crompton, M. M. Gunther, R. F. Ker, and R. M. Alexander. 1999. Dimensions and moment arms of the hind- and forelimb muscles of common chimpanzees (Pan troglodytes). American Journal of Physical Anthropology 110: 179-199.

Tocheri, M. W., and M. W. Marzke. 2007. The recent evolutionary history and adaptive significance of the hominin hand. Journal of Anatomy 210: 771.

Tocheri, M. W., C. M. Orr, M. C. Jacofsky, and M. W. Marzke. 2008. The evolutionary history of the hominin hand since the last common ancestor of Pan and Homo. Journal of Anatomy 212: 544-562.

Tomasello, M., M. Davis-Dasilva, L. Camak, and K. A. Bard. 1987. Observational learning of tool-use by young chimpanzees. Human Evolution 2: 175-183.

Tonooka, R. 2001. Leaf-folding behavior for drinking water by wild chimpanzees (Pan troglodytes verus) at Bossou, Guinea. Animal Cognition 4: 325-334.

Toth, N., and K. Schick. 2009. The Oldowan: The tool making of early hominins and chimpanzees compared. Annual Review of Anthropology 38: 289-305.

Urbani, B., and P. A. Garber. 2002. A stone in their hands ... are monkeys tool users? Anthropologie 40: 183-191.

van Schaik, C. P., A. Ancrenaz, G. Borgen, B. Galdikas, C. D. Knott, I. Singleton, A. Suzuki, S. S. Utami, and M. Merrill. 2003. Orangutan cultures and the evolution of material culture. Science 299: 102-105. 
van Schaik, C. P., R. O. Deaner, and M. Y. Merrill. 1999. The conditions for tool use in primates: Implications for the evolution of material culture. Journal of Human Evolution 36: 719-741.

van Schaik, C. P., E. A. Fox, and A. F. Sitompul. 1996. Manufacture and use of tools in wild Sumatran orangutans: Implications for human evolution. Naturwissenschaften 83: 186-188.

Vignaud, P., P. Duringer, H. T. Mackaye, A. Likius, C. Blondel, J. R. Boisserie, L. de Bonis, V. Eisenmann, M. E. Etienne, D. Geraads, F. Guy, T. Lehmann, F. Lihoreau, N. LopezMartinez, C. Mourer-Chauvire, O. Otero, J. C. Rage, M. Schuster, L. Viriot, A. Zazzo, and M. Brunet. 2002. Geology and palaeontology of the Upper Miocene Toros-Menalla hominid locality, Chad. Nature 418: 152-155.

Villmoare, B., W. H. Kimbel, C. Seyoum, C. J. Campisano, E. N. DiMaggio, J. Rowan, D. R. Braun, J. R. Arrowsmith, and K. E. Reed. 2015. Early Homo at 2.8 Ma from LediGeraru, Afar, Ethiopia. Science 347: 1352-1355.

Visalberghi, E., and D. Fragaszy. 2013. The Etho-Cebus Project: Stone-tool use by wild capuchin monkeys. In C. Sanz, J. Call, and C. Boesch, eds., Tool Use in Animals: Cognition and Ecology, 203-222. Cambridge: Cambridge University Press.

Visalberghi, E., D. Fragaszy, E. Ottoni, P. Izar, M. G. de Oliveira, and F. R. D. Andrade. 2007. Characteristics of hammer stones and anvils used by wild bearded capuchin monkeys (Cebus libidinosus) to crack open palm nuts. American Journal of Physical Anthropology 132: 426-444.

Visalberghi, E., G. Sirianni, D. Fragaszy, and C. Boesch. 2015. Percussive tool use by Taï western chimpanzees and Fazenda Boa Vista bearded capuchin monkeys: A comparison. Philosophical Transactions of the Royal Society B: Biological Sciences 370: 20140351.

Ward, C. V., W. H. Kimbel, E. H. Harmon, and D. C. Johanson. 2012. New postcranial fossils of Australopithecus afarensis from Hadar, Ethiopia (1990-2007). Journal of Human Evolution 63: 1-51.

Ward, C. V., W. H. Kimbel, and D. C. Johanson. 2011. Complete fourth metatarsal and arches in the foot of Australopithecus afarensis. Science 331: 750-753.

White, T. D., B. Asfaw, Y. Beyene, Y. Haile-Selassie, C. O. Lovejoy, G. Suwa, and G. WoldeGabriel. 2009. Ardipithecus ramidus and the paleobiology of early hominids. Science 326: 75-86.

Whiten, A. 2011. The scope of culture in chimpanzees, humans and ancestral apes. Philosophical Transactions of the Royal Society B: Biological Sciences 366: 997-1007.

Whiten, A., J. Goodall, W. C. McGrew, T. Nishida, V. Reynolds, Y. Sugiyama, C. E. G. Tutin, R. W. Wrangham, and C. Boesch. 1999. Cultures in chimpanzees. Nature 399: 682-685.

Whiten, A., K. Schick, and N. Toth. 2009. The evolution and cultural transmission of percussive technology: Integrating evidence from palaeoanthropology and primatology. Journal of Human Evolution 57: 420-435.

Wittiger, L., and J. L. Sunderland-Groves. 2007. Tool use during display behavior in wild Cross River gorillas. American Journal of Primatology 69: 1307-1311. 
WoldeGabriel, G., Y. Haile-Selassie, P. R. Renne, W. K. Hart, S. H. Ambrose, B. Asfaw, G. Heiken, and T. White. 2001. Geology and palaeontology of the Late Miocene Middle Awash valley, Afar rift, Ethiopia. Nature 412: 175-178.

Yamakoshi, G. 1998. Dietary responses to fruit scarcity of wild chimpanzees at Bossou, Guinea: Possible implications for ecological importance of tool use. American Journal of Physical Anthropology 106: 283-295.

Young, N. M., T. D. Capellini, N. T. Roach, and Z. Alemseged. 2015. Fossil hominin shoulders support an African ape-like last common ancestor of humans and chimpanzees. Proceedings of the National Academy of Sciences 112: 11829-11834. 